\title{
Large random simplicial complexes, II; the fundamental group
}

\author{
A. Costa* and M. Farber ${ }^{\dagger}$ \\ School of Mathematical Sciences \\ Queen Mary University of London \\ London E1 $4 \mathrm{NS}$, UK \\ *armindocosta@gmail.com \\ ${ }^{\dagger} M . F a r b e r @ q m u l . a c . u k$
}

Received 13 August 2015

Accepted 20 May 2016

Published 22 June 2016

\begin{abstract}
We study random simplicial complexes in the multi-parameter model focusing mainly on the properties of the fundamental groups. We describe thresholds for nontrivially and hyperbolicity (in the sense of Gromov) for these groups. Besides, we find domains in the multi-parameter space where these groups have 2-torsion. We also prove that these groups never have odd-prime torsion and their geometric and cohomological dimensions are either $0,1,2$ or $\infty$. Another result presented in this paper states that aspherical 2dimensional subcomplexes of random complexes satisfy the Whitehead Conjecture, i.e. all their subcomplexes are also aspherical, with probability tending to one.
\end{abstract}

Keywords: Random simplicial complex; uniform hyperbolically; cohomological and geometric dimensions; the Whitehead Conjecture.

\section{Contents}

1. Introduction 442

2. Subcomplexes of Random Complexes 444

3. Uniform Hyperbolicity 449

4. Topology of Admissible 2-complexes 455

5. Nontriviality of the Fundamental Groups of Random Simplicial Complexes 460

6. The Existence of 2-torsion 463

7. Higher Torsion 469

${ }^{\dagger}$ Corresponding author

This is an Open Access article published by World Scientific Publishing Company. It is distributed under the terms of the Creative Commons Attribution 4.0 (CC-BY) License. Further distribution of this work is permitted, provided the original work is properly cited. 
8. Asphericity and the Whitehead Conjecture

9. Geometric and Cohomological Dimension of the Fundamental Group of a Random Simplicial Complex

Appendix: Proof of Theorem 15

\section{Introduction}

This paper develops the multi-parameter model of random simplicial complexes initiated in [12] and [13].

One of the main motivations to study random simplicial complexes comes from the theory of large networks. Traditionally one model networks by graphs with nodes representing objects and edges representing connections between the objects [22]. However, if we are interested not only in pairwise relations between the objects but also in relations between multiple objects, we may use the high dimensional simplicial complexes instead of graphs as mathematical models of networks.

The mathematical theory of large random simplicial complexes is a new active research area, see [9] and [19] for surveys.

The multi-parameter model which we discuss here allows regimes controlled by a combination of probability parameters associated to various dimensions. This model includes the well-known Linial-Meshulam-Wallach model [20, 21] as an important special case; as another important special case it includes the random simplicial complexes arising as clique complexes of random Erdős-Rényi graphs, see [11, 18].

In the multi-parameter model one starts with a set of $n$ vertices and retains each of them with probability $p_{0}$; on the next step one connects every pair of retained vertices by an edge with probability $p_{1}$, and then fills in every triangle in the obtained random graph with probability $p_{2}$, and so on. As a result we obtain a random simplicial complex depending on the set of probability parameters

$$
\left(p_{0}, p_{1}, \ldots, p_{r}\right), \quad 0 \leq p_{i} \leq 1 .
$$

The topological and geometric properties of multi-parameter random simplicial complexes depend on the whole set of parameters and their thresholds can be understood as subsets of the space of multi-parameter space and not as single numbers as in all the previously studied models.

In our recent paper [13] we described the conditions under which a multiparameter random simplicial complex is connected and simply connected. In [14] we showed that the Betti numbers of multi-parameter random simplicial complexes in one specific dimension dominate significantly the Betti numbers in all other dimensions. In this paper we focus mainly on the properties of fundamental groups of multi-parameter random simplicial complexes, which can be viewed as a new class of random groups. We describe thresholds for nontrivially and hyperbolicity (in the sense of Gromov) for these groups. Besides, we find domains in the multi-parameter space where these groups have 2-torsion. We also prove that these groups have no odd-prime torsion and their geometric and cohomological dimensions are either 0,1 , 
2 or $\infty$. Another result presented in this paper states that aspherical 2-dimensional subcomplexes of random complexes satisfy the Whitehead Conjecture, i.e. all their subcomplexes are also aspherical (with probability tending to one).

To make this paper less dependent on [13] we give now a brief description of the multi-parameter model. Fix an integer $r \geq 0$ and a sequence

$$
\mathfrak{p}=\left(p_{0}, p_{1}, \ldots, p_{r}\right)
$$

of real numbers satisfying

$$
0 \leq p_{i} \leq 1
$$

Denote $q_{i}=1-p_{i}$. We consider the probability space $\Omega_{n}^{r}$ consisting of all subcomplexes $Y \subset \Delta_{n}$ with $\operatorname{dim} Y \leq r$, where the symbol $\Delta_{n}^{(r)}$ stands for the $r$-dimensional skeleton of $\Delta_{n}$, which is defined as the union of all simplexes of dimension $\leq r$. The probability function

$$
\mathbb{P}_{r, \mathfrak{p}}: \Omega_{n}^{r} \rightarrow \mathbb{R}
$$

is given by the formula

$$
\mathbb{P}_{r, \mathfrak{p}}(Y)=\prod_{i=0}^{r} p_{i}^{f_{i}(Y)} \cdot \prod_{i=0}^{r} q_{i}^{e_{i}(Y)} .
$$

Here $e_{i}(Y)$ denotes the number of $i$-dimensional external faces of $Y$, see [13] for the definition. We use the convention $0^{0}=1$; in other words, if $p_{i}=0$ and $f_{i}(Y)=0$ then the corresponding factor in (1) equals 1.

Some results presented in the paper are illustrated by Fig. 1. There for simplicity we assume that the probability parameters $p_{i}$ have the form

$$
p_{i}=n^{-\alpha_{i}} \text {, }
$$

where $\alpha_{i} \geq 0$ are constant. We also assume that $\alpha_{0}=0$ and hence various properties of the fundamental group are described by subsets of the $\left(\alpha_{1}, \alpha_{2}\right)$-plane since they clearly depend only on $p_{1}$ and $p_{2}$. Figure 1 shows domains corresponding to triviality and nontriviality, existence and non-existence of 2-torsion as well as the domain showing when the geometric and cohomological dimension of the fundamental group are $\leq 2$.

In area (a) the complex is connected and simply connected, as proven in [13]. In area (d) the random complex is disconnected and has no cycles and no 2-simplexes, i.e. it is a forest. Of course, all these properties are satisfied asymptotically almost surely (a.a.s.), i.e. the limit of the probability that the corresponding property holds tends to 1 as $n \rightarrow \infty$.

The properties of fundamental groups of random simplicial complexes along the coordinate axis of Fig. 1 were known from earlier published papers and we want to mention the relevant references. The case $\alpha_{1}=0$ corresponds to the LinialMeshulam-Wallach model; in this case the ranges for the triviality and hyperbolicity were established in [2]; the range for 2-torsion as well as the ranges for cohomological 


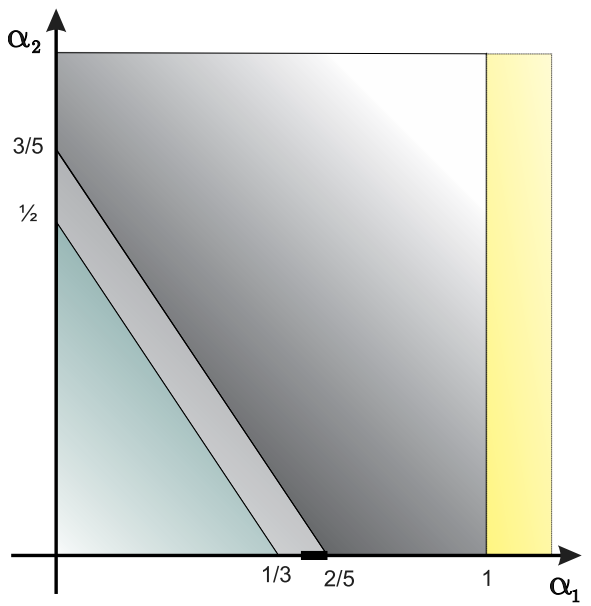

Fig. 1. Areas on the $\left(\alpha_{1}, \alpha_{2}\right)$-plane corresponding to various properties of the fundamental group: (a) light green - the group is trivial; (b) grey - the group has 2-torsion and is hyperbolic; (c) shaded black (including the horizontal interval $(11 / 30,2 / 5)$ shown in bold) - the group is nontrivial, hyperbolic, its geometric dimension is $\leq 2$; (d) yellow - the group is trivial for any choice of the base point.

dimension $1,2, \infty$ were found in [10]; the range when the fundamental group is free was given in [7]. The case when $\alpha_{2}=0$ corresponds to clique complexes of the Erdös-Rényi random graphs. In this case most of the results of Fig. 1 were obtained in [3] and in [11].

\section{Subcomplexes of Random Complexes}

Let $S$ be a fixed simplicial complex of dimension $\leq r$. Similarly to the random graph theory, one may wish to determine conditions when $S$ is embeddable into a random simplicial complex $Y \in \Omega_{n}^{r}$ with respect to the probability measure $\mathbb{P}_{r, \mathfrak{p}}$, where $\mathfrak{p}=\left(p_{0}, p_{1}, \ldots, p_{r}\right)$. In a recent paper [12] we discussed the containment problem under a simplifying assumption that $p_{i}=n^{-\alpha_{i}}$ where $\alpha_{i} \geq 0$ are constant. In this paper we shall need the containment result without this assumption. Besides the general containment result stated as Theorem 1, we also treat in this section the relative containment problem, see Theorem 4 .

Theorem 1. Consider a random simplicial complex $Y \in \Omega_{n}^{r}$ with respect to the measure $\mathbb{P}_{r, \mathfrak{p}}$, where $\mathfrak{p}=\left(p_{0}, p_{1}, \ldots, p_{r}\right)$. Let $S$ be a fixed finite simplicial complex of dimension $\leq r$.

A. Suppose that

$$
n \cdot \min _{T \subset S} \prod_{i=0}^{r} p_{i}^{\frac{f_{i}(T)}{f_{0}(T)}} \rightarrow 0 .
$$

Here $T \subset S$ runs over all nonempty simplicial subcomplexes of $S$ and $f_{i}(T)$ denotes the number of $i$-dimensional faces in $T$. Then the probability that a random 
complex $Y \in \Omega_{n}^{r}$ contains a simplicial subcomplex isomorphic to $S$ tends to zero as $n \rightarrow \infty$.

B. Suppose that for any nonempty subcomplex $T \subset S$ one has

$$
n^{f_{0}(T)} \cdot \prod_{i=0}^{r} p_{i}^{f_{i}(T)} \rightarrow \infty .
$$

Then a random complex $Y \in \Omega_{n}^{r}$ contains a subcomplex isomorphic to $S$ with probability tending to 1 .

Proof. Let us start with the statement A. For $T \subset S$ let $X_{T}: \Omega_{n}^{r} \rightarrow \mathbb{R}$ denote the random variable counting the number of embeddings of $T$ into a random simplicial complex $Y \in \Omega_{n}^{r}$. One has

$$
\mathbb{E}\left(X_{T}\right)=\left(\begin{array}{c}
n \\
f_{0}(T)
\end{array}\right) \cdot f_{0}(T) ! \cdot \prod_{i=0}^{r} p_{i}^{f_{i}(T)} .
$$

Denoting by $\mathbb{P}_{r, \mathfrak{p}}(Y \supset S)$ the probability that $Y$ contains a subcomplex isomorphic to $S$, we have (for $n$ fixed)

$$
\begin{aligned}
\mathbb{P}_{r, \mathfrak{p}}(Y \supset S) & \leq \min _{T \subset S} \mathbb{P}_{r, \mathfrak{p}}(Y \supset T) \leq \min _{T \subset S} \mathbb{E}\left(X_{T}\right) \\
& \leq \min _{T \subset S} n^{f_{0}(T)} \cdot \prod_{i=0}^{r} p_{i}^{f_{i}(T)} .
\end{aligned}
$$

By (2) for any $\epsilon \in(0,1)$ there exists $N$ such that for any $n \geq N$ there exists $T_{n} \subset S$, $T \neq \emptyset$, such that

$$
n \cdot \prod_{i=0}^{r} p_{i}^{\frac{f_{i}\left(T_{n}\right)}{f_{0}\left(T_{n}\right)}}<\epsilon<1 .
$$

Then

$$
n^{f_{0}\left(T_{n}\right)} \cdot \prod_{i=0}^{r} p_{i}^{f_{i}\left(T_{n}\right)}<\epsilon^{f_{0}\left(T_{n}\right)}<\epsilon
$$

which shows that the R.H.S. of (3) tends to zero when $n \rightarrow \infty$ under our assumption (2).

We now prove statement B. We follow the argument used in the proof of Lemma 3.4 from [12]. Let $F_{0}(S)$ denote the set of vertices of $S$ and let $J: F_{0}(S) \rightarrow[n]$ be an embedding. Consider the indicator random variable $X_{J}: \Omega_{n}^{r} \rightarrow\{0,1\}$ taking the value 1 on a subcomplex $Y \in \Omega_{n}^{r}$ iff $J$ extends to a simplicial embedding $J: S \rightarrow Y$. Then $X=\sum_{J} X_{J}$ counts the number of copies of $S$ in $Y$. We wish to show that $X>0$ a.a.s.

We have

$$
\mathbb{E}(X)=(1+o(1)) \cdot n^{f_{0}(S)} \cdot \prod_{i=0}^{r} p_{i}^{f_{i}(S)} \rightarrow \infty
$$


By Chebyshev inequality,

$$
\mathbb{P}(X=0) \leq \frac{\operatorname{Var}(X)}{\mathbb{E}(X)^{2}}
$$

Our goal is to show that $\frac{\operatorname{Var}(X)}{\mathbb{E}(X)^{2}} \rightarrow 0$. One has

$$
\operatorname{Var}(X)=\sum_{J, J^{\prime}} \operatorname{Cov}\left(X_{J}, X_{J^{\prime}}\right)=\sum_{J, J^{\prime}}\left(\mathbb{E}\left(X_{J} X_{J^{\prime}}\right)-\mathbb{E}\left(X_{J}\right) \mathbb{E}\left(X_{J^{\prime}}\right)\right) .
$$

The product $X_{J} \cdot X_{J^{\prime}}$ is the indicator random variable for the containment of the simplicial complex $J(S) \cup J^{\prime}(S)$. Hence we have

$$
\mathbb{E}\left(X_{J} X_{J^{\prime}}\right)=\prod_{i=0}^{r} p_{i}^{2 f_{i}(S)-f_{i}\left(T^{\prime}\right)},
$$

where $T^{\prime}=J(S) \cap J^{\prime}(S)$.

Denote by $T$ the subcomplex $T=J^{-1}\left(T^{\prime}\right) \subset S$. For a fixed subcomplex $T \subset S$ the number of pairs of embeddings $J, J^{\prime}: S \rightarrow \Delta_{n}$ such that $J^{-1}\left(J(S) \cap J^{\prime}(S)\right)=T$ is bounded above by

$$
C_{T} n^{2 f_{0}(S)-f_{0}(T)},
$$

where $C_{T}$ denotes the number of isomorphic copies of $T$ in $S$. We obtain

$$
\begin{aligned}
\operatorname{Var}(X) & \leq \sum_{T \subset S} C_{T} n^{2 f_{0}(S)-f_{0}(T)} \cdot \prod_{i=0}^{r} p_{i}^{2 f_{i}(S)-f_{i}(T)} \\
& \leq C \cdot \mathbb{E}^{2}(X) \cdot \sum_{T \subset S} C_{T} n^{-f_{0}(T)} \cdot\left(\prod_{i=0}^{r} p_{i}^{-f_{i}(T)}\right) \\
& \leq C^{\prime} \cdot \mathbb{E}^{2}(X) \cdot \sum_{T \subset S, T \neq \emptyset} n^{-f_{0}(T)} \prod_{i=0}^{r} p_{i}^{-f_{i}(T)} \\
& =C^{\prime} \cdot \mathbb{E}^{2}(X) \cdot \sum_{T \subset S, T \neq \emptyset}\left[n^{f_{0}(T)} \prod_{i=0}^{r} p_{i}^{f_{i}(T)}\right]^{-1} .
\end{aligned}
$$

It follows that

$$
\frac{\operatorname{Var}(X)}{\mathbb{E}(X)^{2}} \rightarrow 0
$$

since we assume that $n^{f_{0}(T)} \prod_{i=0}^{r} p_{i}^{f_{i}(T)} \rightarrow \infty$ for all non-empty subcomplexes $T \subset S$.

Example 2. Let $C$ be a simplicial loop of length $m \geq 3$. Then for any non-empty subcomplex $T \subset C$ one has $f_{1}(T) \leq f_{0}(T)$ and therefore

$$
n^{f_{0}(T)} \prod_{i=0}^{r} p_{i}^{f_{i}(T)} \geq\left[n p_{0} p_{1}\right]^{f_{0}(T)} .
$$


From Theorem 1 we obtain that a random complex $Y \in \Omega_{n}^{r}$ contains $C$ as a subcomplex assuming that $n p_{0} p_{1} \rightarrow \infty$.

Next we shall consider a relative version of Theorem 1. Suppose that $S_{1} \supset S_{2}$ are two simplicial complexes; we want to describe conditions when a random simplicial complex $Y \in \Omega_{n}^{r}$ admits an embedding $S_{2} \rightarrow Y$ which cannot be extended to an embedding $S_{1} \rightarrow Y$.

By the definition, an embedding of $S_{1}$ into $Y$ is an injective simplicial map $S_{1} \rightarrow Y$.

We denote $f_{i}\left(S_{1}, S_{2}\right)=f_{i}\left(S_{1}\right)-f_{i}\left(S_{2}\right)$ where $i=0, \ldots$

Theorem 3. Assume that for any nonempty subcomplex $T \subset S_{2}$ one has

$$
n^{f_{0}(T)} \cdot \prod_{i=0}^{r} p_{i}^{f_{i}(T)} \rightarrow \infty
$$

and besides,

$$
n^{f_{0}\left(S_{1}, S_{2}\right)} \cdot \prod_{i=0}^{r} p_{i}^{f_{i}\left(S_{1}, S_{2}\right)} \rightarrow 0 .
$$

Then the number of embeddings of $S_{1}$ into a random simplicial complex $Y \in \Omega_{n}^{r}$ is smaller than the number of embeddings of $S_{2}$ into $Y$, a.a.s. In particular, under the assumptions (4), (5) there exists an embedding of $S_{2} \rightarrow Y$ which does not extend to an embedding $S_{1} \rightarrow Y$, a.a.s.

Proof of Theorem 3. For $i=1,2$, let $X_{i}: \Omega_{n}^{r} \rightarrow \mathbb{Z}$ be the random variable that counts the number of embeddings of $S_{i}$ into the random complex $Y \in \Omega_{n}^{r}$. Our goal is to show that $X_{1}<X_{2}$, a.a.s. This would mean that there exists an embedding of $S_{2} \rightarrow Y$ which does not extend to an embedding $S_{1} \rightarrow Y$, a.a.s. We prove below a stronger statement that for any fixed $\epsilon>0$ one has

$$
X_{1}<\epsilon \cdot X_{2}
$$

with probability tending to 1 as $n \rightarrow \infty$.

We have

$$
\frac{\mathbb{E}\left(X_{1}\right)}{\mathbb{E}\left(X_{2}\right)} \leq C \frac{n^{f_{0}\left(S_{1}\right)} \prod_{i=0}^{r} p_{i}^{f_{i}\left(S_{1}\right)}}{n^{f_{0}\left(S_{2}\right)} \prod_{i=0}^{r} p_{i}^{f_{i}\left(S_{2}\right)}}=C \cdot n^{f_{0}\left(S_{1}, S_{2}\right)} \prod_{i=0}^{r} p_{i}^{f_{i}\left(S_{1}, S_{2}\right)} \rightarrow 0
$$

tends to zero because of our assumption (5).

Denote

$$
x_{1}=\frac{\epsilon}{2} \sqrt{\mathbb{E}\left(X_{1}\right) \mathbb{E}\left(X_{2}\right)}
$$

and

$$
\begin{aligned}
x_{2} & =\mathbb{E}\left(X_{2}\right)-\mathbb{E}\left(X_{1}\right)-\sqrt{\mathbb{E}\left(X_{1}\right) \mathbb{E}\left(X_{2}\right)} \\
& =\mathbb{E}\left(X_{2}\right)\left[1-\frac{\mathbb{E}\left(X_{1}\right)}{\mathbb{E}\left(X_{2}\right)}-\sqrt{\frac{\mathbb{E}\left(X_{1}\right)}{\mathbb{E}\left(X_{2}\right)}}\right] .
\end{aligned}
$$


Then

$$
\frac{\mathbb{E}\left(X_{1}\right)}{x_{1}} \rightarrow 0 \text { and } \frac{\mathbb{E}\left(X_{2}\right)}{x_{2}} \rightarrow 1 .
$$

By the Markov inequality we have

$$
\mathbb{P}_{r, n}\left(X_{1} \geq \mathbb{E}\left(X_{1}\right)+x_{1}\right) \leq \frac{\mathbb{E}\left(X_{1}\right)}{\mathbb{E}\left(X_{1}\right)+x_{1}}=\frac{\mathbb{E}\left(X_{1}\right) / x_{1}}{1+\mathbb{E}\left(X_{1}\right) / x_{1}} \rightarrow 0 .
$$

By Chebyschev's inequality

$$
\mathbb{P}_{r, n}\left(X_{2}<\mathbb{E}\left(X_{2}\right)-x_{2}\right)<\frac{\operatorname{Var}\left(\mathrm{X}_{2}\right)}{x_{2}^{2}}=\frac{\operatorname{Var}\left(\mathrm{X}_{2}\right)}{\mathbb{E}\left(X_{2}\right)^{2}} \cdot\left(\frac{\mathbb{E}\left(X_{2}\right)}{x_{2}}\right)^{2} \rightarrow 0
$$

Here we use the fact (established in the proof of Theorem 1) that the assumption (4) implies $\frac{\operatorname{Var}\left(\mathrm{X}_{2}\right)}{\mathbb{E}\left(X_{2}\right)^{2}} \rightarrow 0$.

Thus we see that with probability tending to 1 as $n \rightarrow \infty$ one has the inequalities

$$
\frac{X_{1}}{X_{2}} \leq \frac{\mathbb{E}\left(X_{1}\right)+x_{1}}{\mathbb{E}\left(X_{2}\right)-x_{2}}=\frac{\mathbb{E}\left(X_{1}\right)+\frac{\epsilon}{2} \sqrt{\mathbb{E}\left(X_{1}\right) \mathbb{E}\left(X_{2}\right)}}{\mathbb{E}\left(X_{1}\right)+\sqrt{\mathbb{E}\left(X_{1}\right) \mathbb{E}\left(X_{2}\right)}} \rightarrow \frac{\epsilon}{2} .
$$

Therefore we obtain that

$$
\frac{X_{1}}{X_{2}}<\epsilon
$$

with probability tending to 1 as $n \rightarrow \infty$.

Theorem 4. Let

$$
S_{j} \supset S, \quad j=1, \ldots, N
$$

be a finite family of finite simplicial complexes of dimension $\leq r$ containing a given simplicial complex $S$ and satisfying the following conditions:

(a) for every nonempty subcomplex $T \subset S$ one has

$$
n^{f_{0}(T)} \prod_{i=0}^{r} p_{i}^{f_{i}(T)} \rightarrow \infty
$$

(b) For any $j=1, \ldots, N$,

$$
n^{f_{0}\left(S_{j}, S\right)} \prod_{i=0}^{r} p_{i}^{f_{i}\left(S_{j}, S\right)} \rightarrow 0
$$

Then with probability tending to one, a random simplicial complex $Y \in \Omega_{n}^{r}$ admits an embedding of $S$ which does not extend to an embedding $S_{j} \rightarrow Y$, for every $j=1, \ldots, N$.

Proof. For $j=1, \ldots, N$, let $X_{j}: \Omega_{n}^{r} \rightarrow \mathbb{Z}$ be the random variable that counts the number of embeddings of $S_{j}$ into a random complex $Y \in \Omega_{n}^{r}$. Besides, let $X: \Omega_{n}^{r} \rightarrow \mathbb{Z}$ be the random variable counting the number of embeddings of $S$ into 
a random complex $Y \in \Omega_{n}^{r}$. As in the proof of Theorem 3 (taking $\epsilon=1 / N$ ), we have $X_{j}<\epsilon X$, a.s.s. Therefore,

$$
\sum_{j=1}^{N} X_{j}<X
$$

with probability tending to 1 as $n \rightarrow \infty$. We obtain that with probability tending to one, a random simplicial complex $Y \in \Omega_{n}^{r}$ admits an embedding of $S$ which does not extend to an embedding $S_{j} \rightarrow Y$, for every $j=1, \ldots, N$.

Examples when Theorem 4 can be applied to produce interesting results appear later, see Secs. 5 and 6.

\section{Uniform Hyperbolicity}

In this section we state a theorem about uniform hyperbolically of random simplicial complexes. This theorem plays a crucial role later in this paper.

First we recall the relevant definitions. Let $X$ be a finite simplicial complex. For a simplicial loop in the 1-skeleton $\gamma: S^{1} \rightarrow X^{(1)} \subset X$, we denote by $|\gamma|$ the length of $\gamma$, i.e. the number of edges composing $\gamma$. If $\gamma$ is null-homotopic, $\gamma \sim 1$, we denote by $A_{X}(\gamma)$ the area of $\gamma$, i.e. the minimal number of 2-simplices in any simplicial filling $V$ for $\gamma$. A simplicial filling (or a simplicial Van Kampen diagram) for a loop $\gamma$ is defined as a pair of simplicial maps $S^{1} \stackrel{i}{\rightarrow} V \stackrel{b}{\rightarrow} X$ (where $V$ is a contractible 2-dimensional complex) such that $\gamma=b \circ i$ and the mapping cylinder of $i$ is a disc with boundary $S^{1} \times 0$, see [2].

Next one defines the isoperimetric constant of $X$ :

$$
I(X)=\inf \left\{\frac{|\gamma|}{A_{X}(\gamma)} ; \gamma: S^{1} \rightarrow X^{(1)}, \gamma \sim 1 \text { in } X\right\} \in \mathbb{R}
$$

Clearly $I(X)=I\left(X^{(2)}\right)$, i.e. the isoperimetric constant $I(X)$ depends only on the 2-skeleton $X^{(2)}$. It is well known that the positivity $I(X)>0$ depends only on the fundamental group $\pi_{1}(X)$; in fact, one of the many equivalent definitions of hyperbolicity of discrete groups in the sense of Gromov [16] states that $\pi_{1}(X)$ is hyperbolic iff $I(X)>0$. Clearly, the precise value of $I(X)$ depends on the simplicial structure of $X$. Knowing $I(X)$ (or a lower bound for it) is extremely useful as we shall demonstrate later in this paper.

Below is the main result of this section:

Theorem 5. Consider a random simplicial complex $Y \in \Omega_{n}^{r}$ with respect to the probability measure $\mathbb{P}_{r, \mathfrak{p}}$, where $\mathfrak{p}=\left(p_{0}, \ldots, p_{r}\right), r \geq 2$. Assume that

$$
n p_{0} \rightarrow \infty
$$

and for some $\epsilon>0$,

$$
\left(n p_{0}\right)^{1+\epsilon} p_{1}^{3} p_{2}^{2} \rightarrow 0
$$


Then there exists a constant $c_{\epsilon}>0$ (depending only on $\epsilon$ ) such that a random complex $Y \in \Omega_{n}^{r}$ has the following property with probability tending to one: any subcomplex $Y^{\prime} \subset Y$ satisfies

$$
I\left(Y^{\prime}\right) \geq c_{\epsilon}
$$

In particular, any subcomplex $Y^{\prime} \subset Y$ has a Gromov hyperbolic fundamental group, a.a.s.

Thus, under the assumption (11), all random complexes and their subcomplexes have isoperimetric constants bounded below by $c_{\epsilon}>0$ with probability tending to 1 as $n \rightarrow \infty$.

Assumption (10) guarantees that the number of vertices of $Y$ tends to infinity, see [12], Lemma 2.5 .

Using Theorem 5 we shall establish the following corollary:

Corollary 6. (see Theorem 30) If additionally to the hypothesis of Theorem 5 one has

$$
n p_{0} p_{1} \rightarrow \infty
$$

as $n \rightarrow \infty$ then a random complex $Y \in \Omega_{n}^{r}$ has nontrivial fundamental group, a.a.s.

To illustrate the importance of assumption (13) note that the "alternative" assumption

$$
n p_{0} p_{1} \rightarrow 0
$$

implies (as is easy to show) that a random complex $Y \in \Omega_{n}^{r}$ is at most one dimensional and has no cycles, i.e. it is a forest, a.a.s. In particular, the fundamental group of $Y$ is trivial with any base point, a.a.s. Note also that (14) implies that $Y$ is disconnected, see Lemma 5.4 in [13].

Corollary 7. Assume that $p_{i}=n^{-\alpha_{i}}$, where $\alpha_{i} \geq 0$ are constants, $i=0, \ldots, r$. Then a random complex $Y \in \Omega_{n}^{r}$ has hyperbolic and nontrivial fundamental group for

$$
\begin{aligned}
\alpha_{0}+3 \alpha_{1}+2 \alpha_{2} & >1 \\
\alpha_{0}+\alpha_{1} & <1 .
\end{aligned}
$$

If either $\alpha_{0}+3 \alpha_{1}+2 \alpha_{2}<1$ or $\alpha_{0}+\alpha_{1}>1$ then the fundamental group $\pi_{1}(Y)$ is trivial, a.a.s.

The last part of Corollary 7 is proven in an earlier paper [13].

Figure 2 depicts the domain where the fundamental group of the random complex is nontrivial. Here we assume that $\alpha_{0}=\alpha_{3}=\alpha_{4}=\cdots=0$. 


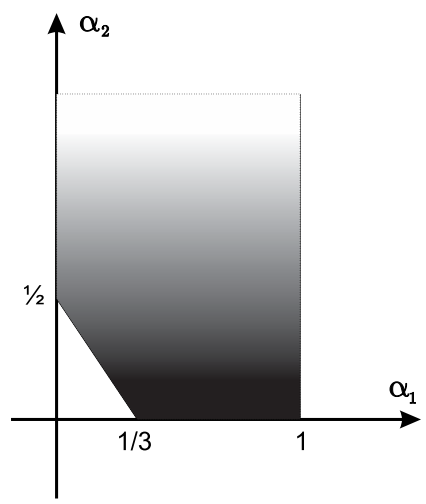

Fig. 2. The region on the plane of $\alpha_{1}, \alpha_{2}$ where the fundamental group $\pi_{1}(Y)$ is nontrivial and hyperbolic in the sense of Gromov.

Next we state the local-to-global principle of Gromov which plays a crucial role in the proof of Theorem 5 . Recall that a 2-complex is said to be pure if every vertex and every edge are incident to a face.

Theorem 8. Let $X$ be a finite 2-complex and let $0<C<11$ be a constant such that any pure subcomplex $S \subset X$ having at most $(44)^{3} \cdot C^{-2} 2$-dimensional simplexes satisfies $I(S) \geq C$. Then $I(X) \geq C \cdot 44^{-1}$.

Theorem 8 follows from [2], Theorem 3.9. Indeed, suppose that the assumptions of Theorem 6.1 are satisfied. Let $\gamma: S^{1} \rightarrow X$ be a simplicial loop with $A_{X}(\gamma)<$ $(44)^{3} \cdot C^{-2}=44 \rho^{2}$, where $\rho=44 / C$. Then there is a pure subcomplex $S \subset X$ with at most $(44)^{3} \cdot C^{-2}$ faces which contains both $\gamma$ and the minimal spanning disk of $\gamma$ in $X$, and by our assumption

$$
\frac{|\gamma|}{A_{S}(\gamma)} \geq I(S) \geq C=44 / \rho
$$

i.e. $A_{X}(\gamma)=A_{S}(\gamma) \leq \frac{\rho}{44} \cdot|\gamma|$. Applying Theorem 3.9 from [2] we obtain $I(X) \geq$ $\rho^{-1}=C / 44$.

Next we give the following definition.

Definition 9. Let $\epsilon>0$ be a positive number. We shall say that a finite 2dimensional simplicial complex $S$ is $\epsilon$-admissible if the following system of linear inequalities

$$
\left\{\begin{array}{l}
3 \alpha_{1}+2 \alpha_{2}>1+\epsilon, \\
\alpha_{1} f_{1}(T)+\alpha_{2} f_{2}(T)<f_{0}(T), \quad T \subset S,
\end{array}\right.
$$

admits a solution with $\alpha_{1} \geq 0, \alpha_{2} \geq 0$, where $T \subset S$ runs over all non-empty subcomplexes. In other words, a 2-complex $S$ is $\epsilon$-admissible if there exist nonnegative real numbers $\alpha_{1}$ and $\alpha_{2}$ such that $3 \alpha_{1}+2 \alpha_{2}>1+\epsilon$ and for any non-empty subcomplex $T \subset S$ one has $\alpha_{1} f_{1}(T)+\alpha_{2} f_{2}(T)<f_{0}(T)$. 
We shall say that a simplicial complex $S$ is admissible if it is $\epsilon$-admissible for some $\epsilon>0$.

The property of being $\epsilon$-admissible is a combinatorial property of a 2-complex $S$ which amounts to certain restrictions on the numbers of vertices, edges and faces for all subcomplexes of $S$. We may mention two special cases:

Case A: A complex $S$ is $\epsilon$-admissible if for any subcomplex $T \subset S$ one has

$$
\mu_{2}(T) \equiv \frac{f_{0}(T)}{f_{2}(T)}>\frac{1}{2}+\frac{\epsilon}{2} .
$$

This is equivalent to Definition 9 under an additional assumption that $\alpha_{1}=0$. Complexes with $\mu_{2}(S)>1 / 2$ were studies in $\S 2$ of [10], see also [2].

Case B: A complex $S$ is $\epsilon$-admissible if for any subcomplex $T \subset S$ one has

$$
\mu_{1}(T) \equiv \frac{f_{0}(T)}{f_{1}(T)}>\frac{1}{3}+\frac{\epsilon}{3} .
$$

This is equivalent to Definition 9 under an additional assumption that $\alpha_{2}=0$. Complexes with $\mu_{1}(S)>1 / 3$ were studies in $\S 5$ of [11].

Example 10. Let $X$ be a simplicial complex $X$ homeomorphic to the torus $T^{2}$. Then using the Euler characteristic relation we obtain $f_{1}(X)=3 f_{0}(X)$ and $f_{2}(X)=$ $2 f_{0}(X)$. The inequality $\alpha_{1} f_{1}(X)+\alpha_{2} f_{2}(X)<f_{0}(X)$ (see the second line in $(15)$ ) is equivalent to $3 \alpha_{1}+2 \alpha_{2}<1$ which contradicts the first line of (15). Hence there is no $\epsilon>0$ such that $X$ is $\epsilon$-admissible.

Remark 11. A 2-complex $S$ is said to be balanced if for any subcomplex $T \subset S$ one has $\mu_{i}(T) \geq \mu_{i}(S)$ for $i=1,2$; see $\S 4$ from [12]. Recall that $\mu_{i}(T)$ denotes the ratio $f_{0}(T) / f_{i}(T)$. A balanced 2-complex $S$ is $\epsilon$-admissible iff

$$
\alpha_{1} f_{1}(S)+\alpha_{2} f_{2}(S)<f_{0}(S)
$$

for some $\alpha_{1} \geq 0$ and $\alpha_{2} \geq 0$ satisfying $3 \alpha_{1}+2 \alpha_{2}>1+\epsilon$. Note that any triangulation of a closed surface with nonnegative Euler characteristic is balanced, see Theorem 4.4 from [12].

Example 12. Let $S$ be a connected 2-complex homeomorphic to a closed surface with positive Euler characteristic, $\chi(S)>0$. Then $S$ is $\epsilon$-admissible assuming that

$$
f_{0}(S) \leq \chi(S) \cdot \frac{1+\epsilon}{\epsilon} .
$$

Indeed, $S$ is balanced (see above) and the well-known Euler type relations imply

$$
f_{1}(S)=3\left(f_{0}(S)-\chi(S)\right), \quad f_{2}(S)=2\left(f_{0}(S)-\chi(S)\right) .
$$

The inequality (16) can be rewritten in this case as

$$
\left(3 \alpha_{1}+2 \alpha_{2}\right) \cdot\left(f_{0}(S)-\chi(S)\right)<f_{0}(S)
$$


and hence the system (15) is equivalent to

$$
1+\epsilon<3 \alpha_{1}+2 \alpha_{2}<\frac{f_{0}(S)}{f_{0}(S)-\chi(S)} .
$$

We see that the existence of $\alpha_{1}, \alpha_{2}$ follows from the inequality

$$
1+\epsilon<\frac{f_{0}(S)}{f_{0}(S)-\chi(S)}
$$

which is equivalent to (17). We obtain that any triangulated sphere or projective plane with sufficiently "small" number of vertices (as prescribed by (17)) is $\epsilon$ admissible.

Therefore, all triangulations of the 2 -sphere and of the real projective plane are admissible.

Example 13. Any graph is $\epsilon$-admissible. Indeed, since $f_{2}(T)=0$, in Definition 9 one may take $\alpha_{1}$ very small and $\alpha_{2}$ very large.

The importance of the notion of $\epsilon$-admissibility stems from the following Lemma:

Lemma 14. Assume that $n p_{0} \rightarrow \infty$ and for some $\epsilon>0$,

$$
\left(n p_{0}\right)^{1+\epsilon} p_{1}^{3} p_{2}^{2} \rightarrow 0 .
$$

For a fixed constant $C>0$, a random simplicial complex $Y \in \Omega_{n}^{r}$ with probability tending to one has the following property: any simplicial pure 2-dimensional subcomplex of $Y$ with at most $C$ 2-simplexes is $\epsilon$-admissible. In other words, under the condition (18), consider the finite set $\mathcal{F}_{C, \epsilon}=\{X\}$ of isomorphism classes of pure 2-dimensional simplicial complexes $X$ satisfying $f_{2}(X) \leq C$ which are not $\epsilon$-admissible. Then with probability tending to 1 a random complex $Y \in \Omega_{n}^{r}$ with respect to the multiparameter measure $\mathfrak{p}=\left(p_{0}, \ldots, p_{r}\right)$ contains none of the complexes $X \in \mathcal{F}_{C, \epsilon}$ as a simplicial subcomplex.

Proof. We may write

$$
p_{i}=n^{-\alpha_{i}} \text {, }
$$

where in general $\alpha_{i}=\alpha_{i}(n)$ is a function of $n$. By our assumption $n p_{0} \rightarrow \infty$, we have $\alpha_{0}(n)<1$ for all large $n$, i.e. for all $n$ except finitely many. Our assumption (18) implies that

$$
3 \alpha_{1}+2 \alpha_{2}-\left(1-\alpha_{0}\right)(1+\epsilon)=\frac{\omega}{\log n},
$$

where $\omega \rightarrow \infty$. This can be rewritten as

$$
3 \alpha_{1}^{\prime}+2 \alpha_{2}^{\prime}=1+\epsilon+x
$$

where

$$
\alpha_{i}^{\prime}=\frac{\alpha_{i}}{1-\alpha_{0}}, \quad x=\frac{\omega}{\left(1-\alpha_{0}\right) \cdot \log n} .
$$


Note that $x \leq 5 \cdot \max \left\{\alpha_{1}^{\prime}, \alpha_{2}^{\prime}\right\}$. For $i=1,2$ define

$$
\beta_{i}(n)= \begin{cases}\alpha_{i}^{\prime}(n)-x / 10, & \text { if } \alpha_{i}^{\prime}(n)=\max \left\{\alpha_{1}^{\prime}(n), \alpha_{2}^{\prime}(n)\right\}, \\ \alpha_{i}^{\prime}(n), & \text { otherwise. }\end{cases}
$$

Then

$$
\beta_{i}(n) \geq 0 \quad \text { and } \quad 3 \beta_{1}(n)+2 \beta_{2}(n)>1+\epsilon
$$

for any $n$.

Let $S$ be a simplicial complex with $f_{2}(S) \leq C$ which is not $\epsilon$-admissible. As follows from Definition 9, for any $n$ there is a subcomplex $T_{n} \subset S$ such that

$$
\beta_{1} f_{1}\left(T_{n}\right)+\beta_{2} f_{2}\left(T_{n}\right) \geq f_{0}\left(T_{n}\right),
$$

which implies that

$$
\alpha_{1}^{\prime} f_{1}\left(T_{n}\right)+\alpha_{2}^{\prime} f_{2}\left(T_{n}\right)-f_{0}\left(T_{n}\right) \geq \frac{x}{10} .
$$

Therefore

$$
\begin{aligned}
\left(n p_{0}\right)^{f_{0}\left(T_{n}\right)} \cdot \prod_{i=1}^{r} p_{i}^{f_{i}\left(T_{n}\right)} & =\left[n^{1-\alpha_{0}}\right]^{f_{0}\left(T_{n}\right)-\sum_{i=1}^{2} \alpha_{i}^{\prime} f_{i}\left(T_{n}\right)} \\
& \leq\left[n^{1-\alpha_{0}}\right]^{-x / 10}=e^{-\omega / 10} .
\end{aligned}
$$

Now we apply Theorem 1 to conclude that the probability that $S$ is embeddable into $Y$ tends to zero as $n \rightarrow \infty$.

A crucial role in the proof of Theorem 5 plays the following theorem stating that all $\epsilon$-admissible 2-dimensional complexes admit a universal lower bound on the value of their isoperimetric constant:

Theorem 15. Given $\epsilon>0$ there exists a constant $C_{\epsilon}>0$ such that for any $\epsilon$ admissible finite simplicial pure 2-complex $X$ one has $I(X) \geq C_{\epsilon}$.

Proof of Theorem 5 using Theorems 8 and 15. Let $C_{\epsilon}>0$ be the constant given by Theorem 15. Consider the set $\mathcal{S}$ of all isomorphism types of finite pure 2-complexes having at most $C=44^{3} \cdot C_{\epsilon}^{-2}$ 2-dimensional simplexes. Clearly, the set $\mathcal{S}$ is finite. Let $\mathcal{S}^{\prime} \subset \mathcal{S}$ denote the subset of complexes in $\mathcal{S}$ which are not $\epsilon$-admissible. Under the assumptions of Theorem 5, a random complex $Y \in \Omega_{n}^{r}$ contains a complex from $\mathcal{S}^{\prime}$ as a subcomplex with probability tending to zero as $n \rightarrow \infty$ as follows from Lemma 14 .

All remaining complexes lying in $\mathcal{S}^{\prime \prime}=\mathcal{S}-\mathcal{S}^{\prime}$ are $\epsilon$-admissible. Theorem 15 states that any complex $S \in \mathcal{S}^{\prime \prime}$ satisfies $I(S) \geq C_{\epsilon}$. Now applying Theorem 8 we obtain that any subcomplex $Y^{\prime} \subset Y$ satisfies $I\left(Y^{\prime}\right) \geq C_{\epsilon} \cdot 44^{-1}=c_{\epsilon}$ with probability tending to 1 as $n \rightarrow \infty$.

Theorem 15 will be proven in the Appendix. 


\section{Topology of Admissible 2-complexes}

In this section we examine the topology of 2-complexes which are admissible in the sense of Definition 9. One of the central results proven here is Theorem 28 describing homotopy types of admissible 2-complexes. In the Appendix we shall continue the study of $\epsilon$-admissible complexes and present a proof of Theorem 15 .

Recall the definitions of the density invariants:

$$
\mu_{i}(S)=\frac{f_{0}(S)}{f_{i}(S)}, \quad i=1,2 .
$$

We shall use the formulas

$$
\mu_{1}(S)=\frac{1}{3}+\frac{3 \chi(S)+L(S)}{3 f_{1}(S)}, \quad \mu_{2}(S)=\frac{1}{2}+\frac{2 \chi(S)+L(S)}{2 f_{2}(S)},
$$

see formula (8) in [11] and formula (2) in [10]. Here

$$
L(S)=\sum_{e}(2-\operatorname{deg} e)
$$

the sum is taken over the edges $e$ of $S$ and for an edge $e$ the symbol $\operatorname{deg} e$ (the degree of $e$ ) denotes the number of 2-simplexes containing $e$.

Lemma 16. Let $S$ be an $\epsilon$-admissible 2-complex. Then for any subcomplex $T \subset S$ either

$$
\mu_{1}(T)>\frac{1+\epsilon}{3} \quad \text { or } \quad \mu_{2}(T)>\frac{1+\epsilon}{2} .
$$

In particular, if $S$ is admissible then for any subcomplex $T \subset S$ one has either

$$
3 \chi(T)+L(T)>0 \quad \text { or } \quad 2 \chi(T)+L(T)>0 .
$$

Moreover, if $S$ is admissible then for any subcomplex $T \subset S$ with $L(T) \leq 0$ one has

$$
\mu_{1}(T)>1 / 3 .
$$

Proof. Suppose that for some $T \subset S$ one has

$$
\mu_{1}(T) \leq(1+\epsilon) / 3 \quad \text { and } \quad \mu_{2}(T) \leq(1+\epsilon) / 2 .
$$

Then for any $\alpha_{1}, \alpha_{2} \geq 0$ satisfying $3 \alpha_{1}+2 \alpha_{2}>1+\epsilon$ one has

$$
\frac{\alpha_{1}}{\mu_{1}(T)}+\frac{\alpha_{2}}{\mu_{2}(T)}>1
$$

which is equivalent to

$$
\alpha_{1} f_{1}(T)+\alpha_{2} f_{2}(T)>f_{0}(T),
$$

implying that $S$ is not $\epsilon$-admissible.

The other statements follow from formula (20).

Corollary 17. Let $S$ be a simplicial 2-complex homeomorphic to a closed surface. If $\chi(S) \leq 0$ then $S$ is not admissible. 
Proof. Applying the previous Lemma 16 with $T=S$ and observing that $L(S)=0$ we have

$$
\mu_{1}(S)=\frac{1}{3}+\frac{\chi(S)}{f_{1}(S)} \leq \frac{1}{3}
$$

which shows that $S$ is not admissible due to Lemma 16.

In Example 12 we showed that any closed surface $S$ with $\chi(S)>0$ is admissible. Recall that a 2-complex $S$ is called closed if every edge $e$ of $S$ is contained in at least two 2-simplexes. Note that for a closed complex $S$ one has $L(S) \leq 0$. A 2-complex $S$ is said to be pure if each vertex and each edge of $S$ are contained in a 2-simplex. A 2-complex $S$ is said to be strongly connected if the space $S-V(S)$ is path-connected; here $V(S)$ denotes the set of vertices of $S$.

Corollary 18. Any closed strongly connected 2-dimensional admissible simplicial complex $S$ with $b_{2}(S)=0$ is either a triangulation of the real projective plane $P^{2}$ or the quotient $Q^{2}$ of a triangulation of $P^{2}$ obtained by identifying two adjacent edges.

Proof. By Lemma 16 we have that $\mu_{1}(S)>1 / 3$. Lemma 5.1 of [11] implies that $S$ is a triangulated projective plane $P^{2}$ or the quotient of a triangulation of $P^{2}$ obtained by identifying two adjacent edges.

Definition 19. A finite simplicial 2-complex $Z$ is said to be a minimal cycle if $b_{2}(Z)=1$ and for any proper subcomplex $Z^{\prime} \subsetneq Z$ one has $b_{2}\left(Z^{\prime}\right)=0$.

Definition 20. A minimal cycle $Z$ is said to be of type A if it does not contain closed proper subcomplexes. Otherwise $Z$ is said to be of type B.

Example 21. We give here examples of minimal cycles. Some details of proofs are left to the reader.

(1) A triangulation of the sphere $S^{2}$ is an admissible minimal cycle of type A, see Example 12.

(2) One also obtains an admissible minimal cycle of type A by starting from a triangulation of $S^{2}$ and identifying two vertices.

However, if one identifies more than two vertices, the obtained minimal cycle is not admissible. [This follows by applying formulas (20) and showing that the complex $S$ obtained from a triangulated sphere by identifying more than 2 vertices satisfies $\chi(S) \leq 0$ and therefore $\mu_{1}(S) \leq 1 / 3$ and $\left.\mu_{2}(S) \leq 1 / 2\right]$.

(3) An admissible minimal cycle of type A is obtained from a triangulation of $S^{2}$ by identifying two adjacent edges.

(4) An example of a minimal cycle of type $\mathrm{B}$ is given by the union $Z=P^{2} \cup D^{2}$ where $P^{2}$ is a triangulation of the real projective plane and $D^{2}$ is a triangulated disc such that $P^{2} \cap D^{2}=\partial D^{2}$ is a non-contractible simple closed loop on $P^{2}$. This minimal cycle is admissible if $f_{1}\left(\partial D^{2}\right) \leq 5$ (compare Lemma 24 below). 
(5) Consider the union $Z=P^{2} \cup P^{2}$ of two real projective planes where the intersection $P^{2} \cap P^{2}$ is a loop non-contractible in each of the projective planes. $Z$ is a minimal cycle of type $\mathrm{B}$ which is not admissible as one observes that $\mu_{1}(Z)<1 / 3$ and $\mu_{2}(Z)<1 / 2$ by applying formula $(20)$.

Remark 22. One can easily see that minimal cycles are closed, strongly connected simplicial complexes. Hence, for any minimal cycle $Z$ one has $L(Z) \leq 0$; besides, $\chi(Z) \leq 2$. Using Lemma 16 we find that every admissible minimal cycle $Z$ must satisfy $3 \chi(Z)+L(Z)>0$ implying that

$$
1 \leq \chi(Z) \leq 2 \text { and } \quad-5 \leq L(Z) \leq 0
$$

Lemma 23. Any admissible minimal cycle $Z$ of type $A$ is homotopy equivalent to either $S^{2}$ or to $S^{2} \vee S^{1}$. Moreover, for every 2-simplex $\sigma \subset Z$ the boundary $\partial \sigma$ is null-homotopic in $Z-\operatorname{Int}(\sigma)$.

Proof. Suppose $Z$ is an admissible minimal cycle of type A. Since $Z$ is closed, one has $L(Z) \leq 0$. Using the second part of Lemma 16, we obtain $\mu_{1}(Z)>1 / 3$. Now we may apply Lemma 5.6 from [11]. Note that the statement of Lemma 5.6 from [11] requires that $\mu_{1}(T)>1 / 3$ for any subcomplex $T \subset Z$; however the proof presented in [11] uses only the assumption $\mu_{1}(Z)>1 / 3$.

Next we establish the following simple fact about minimal cycles of type B which strengthens Remark 22.

Lemma 24. Every admissible minimal cycle $Z$ of type $B$ satisfies $\chi(Z)=2$ and $-5 \leq L(Z) \leq-3$.

Proof. Let $Z^{\prime}$ be a proper closed subcomplex of $Z$. If the graph $\Gamma=Z^{\prime} \cap\left(\overline{Z-Z^{\prime}}\right)$ has no cycles, then

$$
b_{2}(Z)=b_{2}\left(Z^{\prime}\right)+b_{2}\left(\overline{Z-Z^{\prime}}\right)
$$

and either $b_{2}\left(Z^{\prime}\right)=1$ or $b_{2}\left(\overline{Z-Z^{\prime}}\right)=1$; either of these possibilities contradicts the minimality of $Z$. Hence the graph $\Gamma$ must contain a cycle and in particular, the number of edges of $\Gamma$ satisfies $f_{1}(\Gamma) \geq 3$. Each edge of $Z^{\prime}$ is incident to at least two faces of $Z^{\prime}$ and every edge of $\Gamma \subset Z^{\prime}$ is incident to at least one face of $Z$ which is not in $Z^{\prime}$. Since $f_{1}(\Gamma) \geq 3$ and $Z$ is closed it follows that $L(Z) \leq-3$. Since $Z$ is admissible, by Lemma 16 we obtain $0<3 \chi(Z)+L(Z) \leq 3 \chi(Z)-3$. In particular, $\chi(Z)>1$. Since $b_{0}(Z)=b_{2}(Z)=1$ we obtain $\chi(Z)=2$, as claimed.

Definition 25. Let $Z$ be an admissible minimal cycle of type B. Any closed strongly connected proper subcomplex $Z_{0} \subset Z$ is called a core of $Z$. 
Clearly, for any core $Z_{0} \subset Z$ one has $b_{2}\left(Z_{0}\right)=0$ (by minimality). Applying Corollary 18 we see that any core is homeomorphic either to $P^{2}$ or to $P^{2}$ with two adjacent edges identified.

Lemma 26. An admissible minimal cycle $Z$ of type $B$ has a unique core $Z_{0} \subset Z$.

Proof. Let $Z$ be an admissible minimal cycle of type B. Let us assume that $Z$ has two distinct cores $Z^{\prime}, Z^{\prime \prime} \subset Z$.

Consider the graphs

$$
\Gamma^{\prime}=Z^{\prime} \cap \overline{Z-Z^{\prime}}, \quad \Gamma^{\prime \prime}=Z^{\prime \prime} \cap \overline{Z-Z^{\prime \prime}} .
$$

By the arguments used in the proof of Lemma 24 we obtain that $f_{1}\left(\Gamma^{\prime}\right) \geq 3$ and $f_{1}\left(\Gamma^{\prime \prime}\right) \geq 3$. The graphs $\Gamma^{\prime}$ and $\Gamma^{\prime \prime}$ cannot be edge-disjoint since otherwise $Z$ would have at least 6 edges of degree $\geq 3$; the latter would give $L(Z) \leq-6$ contradicting Lemma 24. This implies that $f_{1}\left(Z^{\prime} \cap Z^{\prime \prime}\right)>0$ and therefore the union $Z^{\prime} \cup Z^{\prime \prime}$ is strongly connected.

We observe next that the union $Z^{\prime} \cup Z^{\prime \prime}$ must coincide with $Z$. Indeed, if $Z^{\prime} \cup$ $Z^{\prime \prime} \neq Z$ then by minimality $b_{2}\left(Z^{\prime} \cup Z^{\prime \prime}\right)=0$ and by Corollary 18 the union $Z^{\prime} \cup Z^{\prime \prime}$ is either homeomorphic to $P^{2}$ or to the quotient $Q^{2}$ of $P^{2}$ where two adjacent edges are identified. But neither $P^{2}$ nor $Q^{2}$ admits a triangulation in which it is a union of two distinct closed proper subcomplexes. Here we use our assumption $Z^{\prime} \neq Z^{\prime \prime}$.

Now we may show that

$$
\Gamma^{\prime}=S \cap \overline{Z^{\prime \prime}-S}, \quad \Gamma^{\prime \prime}=S \cap \overline{Z^{\prime}-S},
$$

where

$$
S=Z^{\prime} \cap Z^{\prime \prime}
$$

is the intersection. Indeed, since $Z=Z^{\prime} \cup Z^{\prime \prime}$ one obtains $Z-Z^{\prime}=Z^{\prime \prime}-S$ and

$$
\begin{aligned}
\Gamma^{\prime} & =Z^{\prime} \cap \overline{Z-Z^{\prime}} \\
& =Z^{\prime} \cap \overline{Z^{\prime \prime}-S} \\
& =\left[S \cap \overline{Z^{\prime \prime}-S}\right] \cup\left[\left(Z^{\prime}-S\right) \cap \overline{Z^{\prime \prime}-S}\right] \\
& =S \cap \overline{Z^{\prime \prime}-S .} .
\end{aligned}
$$

On the last step we used the observation $\left(Z^{\prime}-S\right) \cap \overline{Z^{\prime \prime}-S} \subset\left(Z^{\prime}-S\right) \cap Z^{\prime \prime}=\emptyset$. The statement regarding $\Gamma^{\prime \prime}$ follows similarly.

We know that $\chi\left(Z^{\prime} \cup Z^{\prime \prime}\right)=2$ and $\chi\left(Z^{\prime}\right)=\chi\left(Z^{\prime \prime}\right)=1$; therefore $\chi(S)=0$. If $S$ is disconnected then $b_{1}(Z)=b_{1}\left(Z^{\prime} \cup Z^{\prime \prime}\right) \geq 1$ contradicting $\chi(Z)=2$. Hence we obtain

$$
b_{0}(S)=b_{1}(S)=1 .
$$

Suppose that the intersection $S$ has no 2-faces. Then $S$ is a connected graph and every edge of $S$ has degree $\geq 4$ in $Z$ since it is incident to at least two faces of 
$Z^{\prime}$ and two faces of $Z^{\prime \prime}$. However, since $b_{1}(S)=1$ we have $f_{1}(S) \geq 3$ implying that $L(Z) \leq-2 f_{1}(S) \leq-6$; this contradicts $L(Z) \geq-5$, see above.

Thus we see that $f_{2}(S) \geq 1$, i.e. the complex $S$ is 2-dimensional.

Note that $b_{1}\left(Z^{\prime}\right)=b_{2}\left(Z^{\prime}\right)=0$ and $b_{1}\left(Z^{\prime \prime}\right)=b_{2}\left(Z^{\prime \prime}\right)=0$, see Corollary 18 . The Mayer-Vietoris exact sequences with rational coefficients for the covers $Z^{\prime}=$ $S \cup \overline{Z^{\prime}-S}$ and $Z^{\prime \prime}=S \cup \overline{Z^{\prime \prime}-S}$ give the isomorphims

$$
\begin{aligned}
H_{1}\left(\Gamma^{\prime} ; \mathbb{Q}\right) \cong H_{1}(S ; \mathbb{Q}) \oplus H_{1}\left(\overline{Z^{\prime \prime}-S} ; \mathbb{Q}\right), \\
H_{1}\left(\Gamma^{\prime \prime} ; \mathbb{Q}\right) \cong H_{1}(S ; \mathbb{Q}) \oplus H_{1}\left(\overline{Z^{\prime}-S} ; \mathbb{Q}\right) .
\end{aligned}
$$

Since $H_{1}(S ; \mathbb{Q})=\mathbb{Q}$ we obtain from $(24),(25)$ :

$$
b_{1}\left(\Gamma^{\prime}\right), \quad b_{1}\left(\Gamma^{\prime \prime}\right) \geq 1 .
$$

In the beginning of the proof we have observed that every edge of $\Gamma^{\prime}$ and of $\Gamma^{\prime \prime}$ has degree $\geq 3$ in $Z$ and that $\Gamma^{\prime}$ and $\Gamma^{\prime \prime}$ cannot be edge-disjoint. Hence $f_{1}\left(\Gamma^{\prime} \cup \Gamma^{\prime \prime}\right) \leq$ 5 and therefore $b_{1}\left(\Gamma^{\prime} \cup \Gamma^{\prime \prime}\right) \leq 2$ since any graph on at most five edges has at most 2 independent cycles. Let us show that the case $b_{1}\left(\Gamma^{\prime} \cup \Gamma^{\prime \prime}\right)=2$ is impossible. If $b_{1}\left(\Gamma^{\prime} \cup \Gamma^{\prime \prime}\right)=2$ then $\Gamma^{\prime} \cup \Gamma^{\prime \prime}$ is a square with one diagonal, it has 5 edges and each of the edges has degree 3 in $Z$ (since $L(Z) \geq-5$, see above). Moreover, in this case all other edges of $Z$ have degree 2 (as again follows from $L(Z) \geq-5$ ). Let $v$ be one of the vertices of degree 3 in the graph $\Gamma^{\prime} \cup \Gamma^{\prime \prime}$. Then $v$ is incident to exactly three odd degree edges in $Z$ (see similar argument in [11], proof of Lemma 5.7 on p. 15). All edges of $Z$ incident to $v$ which do not belong to $\Gamma^{\prime} \cup \Gamma^{\prime \prime}$ have degree 2 in $Z$. In particular, the link $\operatorname{Lk}_{Z}(v)$ of $v$ in $Z$ would be a graph with an odd number of odd degree vertices which is impossible. Therefore, we obtain that

$$
b_{1}\left(\Gamma^{\prime} \cup \Gamma^{\prime \prime}\right)=1 \text {. }
$$

From (26) and (27) it follows that

$$
b_{1}\left(\Gamma^{\prime} \cap \Gamma^{\prime \prime}\right)=b_{1}\left(\Gamma^{\prime}\right)=b_{1}\left(\Gamma^{\prime \prime}\right)=1,
$$

i.e. the graphs $\Gamma^{\prime}$ and $\Gamma^{\prime \prime}$ possess a common cycle $C$. The isomorphisms (24) and (25) give

$$
b_{1}\left(\overline{Z^{\prime}-S}\right)=b_{1}\left(\overline{Z^{\prime \prime}-S}\right)=0 .
$$

We obtain that some integral multiple of the cycle $C$ bounds a $\mathbb{Z}$-chains in $\overline{Z^{\prime}-S}$ and in $\overline{Z^{\prime \prime}-S}$ and the difference of these two chains will be a nontrivial 2dimensional cycle $c$ lying in $Z-S^{\prime} \neq Z$ contradicting the minimality of $Z$. Here $S^{\prime}$ denotes the union of interiors of all 2-simplexes of $S$. Note that the complexes $\overline{Z^{\prime}-S}, \overline{Z^{\prime \prime}-S}$ and $S$ have no common 2-simplexes. This completes the proof.

Lemma 27. Any admissible minimal cycle $Z$ of type $B$ is homotopy equivalent to the sphere $S^{2}$. Let $Z_{0} \subset Z$ denote the core of $Z$. Then for any 2-simplex $\sigma \subset Z_{0}$ the complement $Z-\operatorname{Int}(\sigma)$ is contractible. 
Proof. Consider the complex $Z-\operatorname{Int}(\sigma)$ where $\sigma$ is a 2 -simplex, $\sigma \subset Z_{0}$ lying in the core. Starting from the complex $Z-\operatorname{Int}(\sigma)$ and collapsing subsequently faces across the free edges we shall arrive at a connected graph $\Gamma$, as follows from the uniqueness of the core (Lemma 26). Since $\chi(Z)=2$ (see Lemma 24), we find $\chi(\Gamma)=\chi(Z-\operatorname{Int}(\sigma))=1$. Therefore $\Gamma$ is a tree. Hence the complex $Z-\operatorname{Int}(\sigma)$ is contractible. This implies that $Z$ is homotopy equivalent to the result of attaching a 2 -cell to $Z-\operatorname{Int}(\sigma)$, hence $Z \simeq S^{2}$.

Theorem 28. Any admissible 2-complex $X$ is homotopy equivalent to a wedge of circles, spheres and projective planes.

Proof. We will act by induction on $b_{2}(X)$. If $b_{2}(X)=0$ and $X$ is admissible then using Corollary 18 we see that each strongly connected component of $X$ is homotopy equivalent to $P^{2}$. Hence $X$ is homotopy equivalent to a wedge of circles and projective planes.

Assume now that the statement of the theorem has been proven for all connected admissible 2-complexes $X$ with $b_{2}(X)<k$. Consider an admissible 2-complex $X$ satisfying $b_{2}(X)=k>0$. Find a minimal cycle $Z \subset X$ and observe that the homomorphism $H_{2}(Z ; \mathbb{Z})=\mathbb{Z} \rightarrow H_{2}(X ; \mathbb{Z})$ induced by the inclusion is injective. Let $\sigma \subset Z$ be a 2-simplex; if $Z$ is of type B we shall assume that $\sigma$ lies in the core $Z_{0} \subset Z$. We shall use Lemma 23 or 27 depending on whether $Z$ is of type $\mathrm{A}$ or $\mathrm{B}$. The complex $X^{\prime}=X-\operatorname{Int}(\sigma)$ satisfies $b_{2}\left(X^{\prime}\right)=k-1$ and is admissible and thus by induction $X^{\prime}$ is homotopy equivalent to a wedge of circles, spheres and projective planes. Therefore, $X$ is homotopy equivalent to $X^{\prime} \vee S^{2}$ and hence $X$ is homotopy equivalent to a wedge of circles, spheres and projective planes.

Corollary 29. The fundamental group of any admissible 2-complex $X$ is the free product of several copies of $\mathbb{Z}$ and $\mathbb{Z}_{2}$. In particular, $\pi_{1}(X)$ is hyperbolic.

Corollary 29 is in some sense a weak version of Theorem 15 which will be proven later in the Appendix.

\section{Nontriviality of the Fundamental Groups of Random Simplicial Complexes}

Theorem 30. Let $Y \in \Omega_{n}^{r}$ be a random simplicial complex with respect to the probability measure $\mathbb{P}_{r, \mathfrak{p}}$ where $\mathfrak{p}=\left(p_{0}, p_{1}, \ldots, p_{r}\right)$. Assume that

$$
n p_{0} p_{1} \rightarrow \infty
$$

and for some $\epsilon>0$,

$$
\left(n p_{0}\right)^{1+\epsilon} p_{1}^{3} p_{2}^{2} \rightarrow 0
$$


Then for some choice of the base point $y_{0} \in Y$ the fundamental group $\pi_{1}\left(Y, y_{0}\right)$ is nontrivial, a.a.s.

Remark 31. If the assumption (29) is replaced by the stronger assumption

$$
n p_{0} p_{1}-\log \left(n p_{0}\right) \rightarrow \infty,
$$

then $Y \in \Omega_{n}^{r}$ is connected, a.a.s. (see Corollary 7.2 from [13]). However under the assumption (29) a random complex might be disconnected, see $\S 7$ from [13]) and thus, the fundamental group $\pi_{1}\left(Y, y_{0}\right)$ might depend on the choice of the base point $y_{0} \in Y$.

Remark 32. In the special case when

$$
p_{i}=n^{-\alpha_{i}}
$$

with $\alpha_{i} \geq 0$ constant, where $i=0,1, \ldots$ Theorem 30 states that a random complex $Y$ has a nontrivial fundamental group assuming that

$$
\begin{aligned}
\alpha_{0}+\alpha_{1} & <1, \\
\alpha_{0}+3 \alpha_{1}+2 \alpha_{2} & >1 .
\end{aligned}
$$

From [13] we know that the inequality $\alpha_{0}+\alpha_{1}<1$ implies connectivity of $Y$.

Proof of Theorem 30. Let $C$ be the simplicial loop of length 4, i.e. the boundary of the square. Using Example 2, we note that our assumption (29) implies that a random complex $Y \in \Omega_{n}^{r}$ contains $C$ as a subcomplex with probability tending to one.

We want to show that $Y$ contains $C$ as "an essential subcomplex", i.e. such that the inclusion $C \subset Y$ induces a nontrivial homomorphism $\pi_{1}\left(C, y_{0}\right) \rightarrow \pi_{1}\left(Y, y_{0}\right)$. This would imply that $\pi_{1}\left(Y, y_{0}\right) \neq 1$ for some choice of the base point. We shall use Theorem 4 to show the existence of essential embeddings $C \rightarrow Y$.

Let $c_{\epsilon}>0$ be the constant given by Theorem 5 .

The arguments of the proof which is presented below may seem formal, and to illustrate them we give in this paragraph a brief vague intuitive description. If an inclusion $C \subset Y$ is not essential, then $C \rightarrow Y$ can be extended to a simplicial map $D \rightarrow Y$ where $D$ is a simplicial disc. Using the inequality $I(Y) \geq c_{\epsilon}$ (which we may assume to be satisfied due to Theorem 15), we may assume that $D$ has at most $4 \cdot c_{\epsilon}^{-1} 2$-simplexes. Of course the map $D \rightarrow Y$ does not have to be injective; therefore the image of $D \rightarrow Y$ is a subcomplex $S \subset Y$ with at most $4 \cdot c_{\epsilon}^{-1} 2$ simplexes. There are finitely many isomorphism types of disc triangulations with at most $4 \cdot c_{\epsilon}^{-1} 2$-simplexes and there are finitely many isomorphism types of their simplicial images $S$; those $S$ 's which are not $\epsilon$-admissible appear with probability tending to 0 (due to Lemma 14). Below we formalize the properties of complexes $S$ which may appear in this way and use Theorem 4 to show that there exists an embedding $C \rightarrow Y$ which cannot be extended to an embedding $S \rightarrow Y$; this embedding is clearly essential. 
Now we continue with the formal argument. Consider the set $\mathcal{L}_{\epsilon}$ of isomorphism types of pairs $(S, C)$ where $S$ is a finite 2-complex such that:

(a) the inclusion $C \rightarrow S$ induces the trivial homomorphism of the fundamental groups;

(b) $S$ is minimal in the sense that for any proper subcomplex $C \subset S^{\prime} \subsetneq S$ the inclusion $C \rightarrow S^{\prime}$ induces a nontrivial homomorphism $\pi_{1}(C) \rightarrow \pi_{1}\left(S^{\prime}\right)$;

(c) $f_{2}(S) \leq 4 \cdot c_{\epsilon}^{-1}$;

(d) $S$ is $\epsilon$-admissible.

Note that for $S \in \mathcal{L}_{\epsilon}$ one has $b_{2}(S)=0$. Indeed, if $b_{2}(S) \neq 0$ then $S$ would contain a minimal cycle $Z \subset S$ and this minimal cycle would be $\epsilon$-admissible. By Lemmas 23 and 27 the complex $Z$ contains a 2 -simplex $\sigma$ such that the boundary $\partial \sigma$ is nullhomotopic in $Z-\operatorname{Int}(\sigma)$. Hence removing $\sigma$ does not change the fundamental group and we obtain contradiction with the minimality property (b).

We obtain that $\chi(S) \leq 1$ for any $S \in \mathcal{L}_{\epsilon}$.

It is easy to see that $S$ cannot have edges of degree zero (i.e. $S$ must be pure) and any edge of $S$ of degree 1 must lie in $C$ (as follows from the minimality property (b)). Therefore $L(S) \leq f_{1}(C)=4$.

We observe that for any $S \in \mathcal{L}_{\epsilon}$ one has

$$
\begin{aligned}
& f_{1}(S, C) \geq 3 f_{0}(S, C)+1, \\
& f_{2}(S, C) \geq 2 f_{0}(S, C)+2 .
\end{aligned}
$$

Recall that the notation $f_{i}(S, C)$ stands for $f_{i}(S)-f_{i}(C)$, the number of $i$-simplexes lying in $S-C$. For any simplicial complex $X$ of dimension $\leq 2$ one has

$$
\begin{aligned}
& 3 \chi(X)+L(X)=3 f_{0}(X)-f_{1}(X), \\
& 2 \chi(X)+L(X)=2 f_{0}(X)-f_{2}(X) .
\end{aligned}
$$

Applying these equalities to $S$ and to $C$ and using

$$
\chi(S) \leq 1, \quad L(S) \leq 4, \quad \chi(C)=0, \quad L(C)=8
$$

gives (32) and (33).

Now, using (32) and (33) for $S \in \mathcal{L}_{\epsilon}$ we obtain

$$
n^{f_{0}(S, C)} \prod_{i=0}^{2} p_{i}^{f_{i}(S, C)} \leq\left[n p_{0} p_{1}^{3} p_{2}^{2}\right]^{f_{0}(S, C)} \cdot p_{1} p_{2}^{2} .
$$

We claim that our assumptions (29) and (30) imply that $n p_{0} p_{1}^{3} p_{2}^{2} \rightarrow 0$ and $p_{1} p_{2}^{2} \rightarrow 0$. Indeed, (29) implies that $n p_{0} \rightarrow \infty$ and then (30) implies that $n p_{0} p_{1}^{3} p_{2}^{2} \rightarrow 0$. It is easy to see that $p_{1} p_{2}^{2} \rightarrow 0$ follows.

Next we observe that the set $\mathcal{L}_{\epsilon}$ is finite and hence we may apply Theorem 4 . Since all the assumptions of this theorem are satisfied we obtain that with probability tending to one, for $Y \in \Omega_{n}^{r}$ there exists an embedding $C \rightarrow Y$ which cannot be extended to an embedding $S \rightarrow Y$ for any $S \in \mathcal{L}_{\epsilon}$. 
Consider the set $\Omega_{n}^{\prime} \subset \Omega_{n}^{r}$ consisting of complexes $Y \in \Omega_{n}^{r}$ satisfying the following three conditions:

(1) every subcomplex $Y^{\prime} \subset Y$ satisfies $I\left(Y^{\prime}\right) \geq c_{\epsilon}$;

(2) any 2-dimensional pure subcomplex $S \subset Y$ with $f_{2}(S) \leq 4 \cdot c_{\epsilon}^{-1}$ is $\epsilon$-admissible.

(3) $Y$ contains $C$ as a subcomplex such that no complex $S \in \mathcal{L}_{\epsilon}$ can be embedded into $Y$ extending the embedding $C \rightarrow Y$.

By Theorem 15, Lemma 14, Theorem 4 and Example 2, the probability that $Y$ belongs to $\Omega_{n}^{\prime}$ tends to one as $n \rightarrow \infty$. We show below that for every $Y \in \Omega_{n}^{\prime}$ the fundamental group $\pi_{1}\left(Y, y_{0}\right)$ is nontrivial for some choice of the base point.

Let us explain that for $Y \in \Omega_{n}^{\prime}$ the embedding $C \rightarrow Y$ is essential. For any embedding $C \subset Y$ which is not essential there exists a simplicial disc $D$ with at most $4 \cdot c_{\epsilon}^{-1} 2$-simplexes and $\partial D=C$ which can be simplicially mapped into $Y$ extending the embedding $C \rightarrow Y$ (this follows from the inequality $I(Y) \geq c_{\epsilon}^{-1}$ given by Theorem 5). The image of this disc is a subcomplex $S \subset Y$ containing $C$ which satisfies the properties (a), (c). Property (d) is satisfied because of Lemma 14. If the minimality property (b) is not satisfied, then instead of $S$ we can consider an appropriate smaller subcomplex. Hence any non-essential embedding $C \rightarrow Y$ can be extended to an embedding $S \rightarrow Y$ for some $S \in \mathcal{L}_{\epsilon}$. This completes the proof.

Remark 33. In the proof presented above we showed the existence of essential loops of length 4 . The same argument applies for loops of any fixed length $\geq 4$; it also applies to loops of length 3 under an additional assumption $p_{2} \rightarrow 0$. It is obvious that in the case when $p_{2}=1$ every loop of length 3 is the boundary of a 2-simplex in $Y$ and hence our statement would be false for loops of length 3 .

\section{The Existence of 2-torsion}

Theorem 34. Let $Y \in \Omega_{n}^{r}$ be a random simplicial complex with respect to the probability measure $\mathbb{P}_{r, \mathfrak{p}}$ where $\mathfrak{p}=\left(p_{0}, p_{1}, \ldots, p_{r}\right)$. Assume that

$$
\begin{aligned}
p_{2} & \rightarrow 0, \\
n p_{0} p_{1}^{5 / 2} p_{2}^{5 / 3} & \rightarrow \infty,
\end{aligned}
$$

and for some $\epsilon>0$,

$$
\left(n p_{0}\right)^{1+\epsilon} p_{1}^{3} p_{2}^{2} \rightarrow 0 .
$$

Then the fundamental group $\pi_{1}(Y)$ has elements of order 2, a.a.s.

Remark 35. The assumptions of Theorem 34 imply that $Y \in \Omega_{n}^{r}$ connected, a.a.s. To show this one may use Corollary 7.2 from [13], which requires that $\omega \rightarrow \infty$ and $\omega p_{1}-\log (\omega) \rightarrow \infty$ where $\omega=n p_{0}$. By (36) we have $n^{2 / 5} p_{0}^{2 / 5} p_{1}=\omega^{\prime} \rightarrow \infty$. Hence $\omega p_{1}-\log \omega=\omega^{3 / 5} \cdot \omega^{\prime}-\log \omega \rightarrow \infty$. 
Remark 36. In the special case when

$$
p_{i}=n^{-\alpha_{i}}
$$

with $\alpha_{i} \geq 0$ constant, where $i=0,1, \ldots$ Theorem 34 states that the fundamental group of a random complex $Y$ has a nontrivial element of order 2 assuming that

$$
\begin{aligned}
\alpha_{0}+\frac{5}{2} \alpha_{1}+\frac{5}{3} \alpha_{2} & <1, \\
\alpha_{0}+3 \alpha_{1}+2 \alpha_{2} & >1, \\
\alpha_{2} & >0 .
\end{aligned}
$$

This result was proven in [10] in the special case when $\alpha_{1}=0$. See Fig. 3.

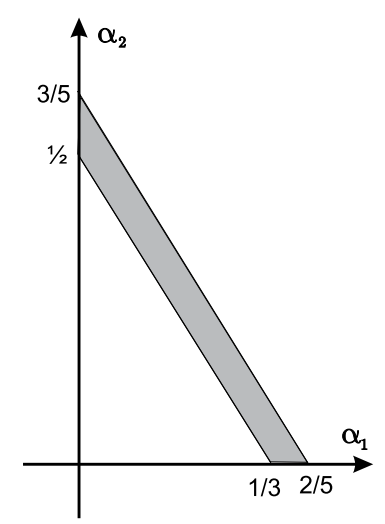

Fig. 3. The region on the plane of $\alpha_{1}, \alpha_{2}$ where the fundamental group $\pi_{1}(Y)$ has 2-tosion.

Proof of Theorem 34. Consider the triangulation $S_{0}$ of the real projective plane $P^{2}$ shown in Fig. 4; it has 6 vertices, 15 1-simplexes and 102 -simplexes. It is the triangulation of $P^{2}$ having the smallest number of vertices. By Theorem 4.4 from [12] $S_{0}$ is balanced, which means that for any non-empty subcomplex $T \subset S_{0}$ one has

$$
\frac{f_{i}(T)}{f_{0}(T)} \leq \frac{f_{i}\left(S_{0}\right)}{f_{0}\left(S_{0}\right)} .
$$

Applying Theorem 1, B, we see that for any $T \subset S_{0}, T \neq \emptyset$,

$$
\begin{aligned}
{\left[n^{f_{0}(T)} \prod_{i=0}^{2} p_{i}^{f_{i}(T)}\right]^{\frac{1}{f_{0}(T)}} } & \geq n \cdot \prod_{i=0}^{2} p_{i}^{\frac{f_{i}(T)}{f_{0}(T)}} \\
& \geq n \cdot \prod_{i=0}^{2} p_{i}^{\frac{f_{i}\left(S_{0}\right)}{f_{0}\left(S_{0}\right)}}=n p_{0} p_{1}^{5 / 2} p_{2}^{5 / 3} \rightarrow \infty .
\end{aligned}
$$

Thus we see that under the assumption (36) the simplicial complex $S_{0}$ embeds into a random complex $Y \in \Omega_{n}^{r}$, a.a.s. 


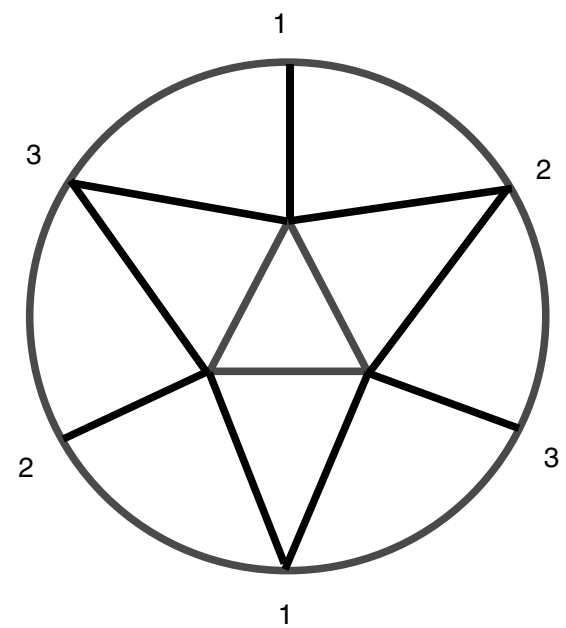

Fig. 4. The triangulation of the real projective plane having 6 vertices, 15 edges and 10 faces. The vertices and edges on the outer circle must be identified as indicated.

We want to show that $Y$ contains $S_{0}$ as "an essential subcomplex", i.e. such that the inclusion $S_{0} \subset Y$ induces a nontrivial homomorphism $\pi_{1}\left(S_{0}\right)=\mathbb{Z}_{2} \rightarrow \pi_{1}(Y)$; this would imply that $\pi_{1}(Y)$ has 2 -torsion. We shall use Theorem 4 to show the existence of an essential embedding $S_{0} \rightarrow Y$. Our strategy will be similar to those used in the proof of Theorem 30 .

We construct below a finite list $\mathcal{L}_{\epsilon}$ of 2-complexes $S$ containing $S_{0}$ such that every non-essential embedding of $S_{0}$ into $Y \in \Omega_{n}^{\prime}$ extends to an embedding $S \rightarrow Y$, for some $S \in \mathcal{L}_{\epsilon}$.

Consider the set $\mathcal{L}_{\epsilon}$ of isomorphism types of pairs $\left(S, S_{0}\right)$ where $S$ is a finite 2-complex containing $S_{0}$ satisfying the following conditions:

(a) the inclusion $S_{0} \rightarrow S$ induces the trivial homomorphism of the fundamental groups;

(b) $S$ is minimal in the sense that for any proper subcomplex $S_{0} \subset S^{\prime} \subsetneq S$ the inclusion $S_{0} \rightarrow S^{\prime}$ induces an injective homomorphism $\pi_{1}\left(S_{0}\right) \rightarrow \pi_{1}\left(S^{\prime}\right)$;

(c) $f_{2}(S) \leq 3 \cdot c_{\epsilon}^{-1}+10$;

(d) $S$ is $\epsilon$-admissible.

Note that any $S \in \mathcal{L}_{\epsilon}$ is pure. Indeed, if $S^{\prime} \subset S$ denotes the pure part of $S$ then $\pi_{1}\left(S^{\prime}\right) \rightarrow \pi_{1}(S)$ is injective and hence the inclusion $S_{0} \subset S^{\prime}$ induces a trivial homomorphism $\pi_{1}\left(S_{0}\right) \rightarrow \pi_{1}\left(S^{\prime}\right)$; therefore the minimality property (b) implies $S=S^{\prime}$.

Consider the minimal cycles contained in a complex $S \in \mathcal{L}_{\epsilon}$. If $Z \subset S$ is a minimal cycle of type A then $Z$ cannot be contained in $S_{0}$ and by Lemma 23 there is a 2 -simplex $\sigma \subset Z-S_{0}$ with $\partial \sigma$ null-homotopic in $Z-\operatorname{Int}(\sigma)$. Removing $\sigma$ from $S$ does not change the fundamental group and leads to a proper subcomplex 
$S_{0} \subset S^{\prime} \subset S$ contradicting the minimality property (b). Hence $S$ does not contain minimal cycles of type A.

Let $Z \subset S$ be a minimal cycle of type B where $S \in \mathcal{L}_{\epsilon}$. Let $Z_{0} \subset Z$ be the core of $Z$. Recall that the core $Z_{0}$ is either homeomorphic to $P^{2}$ or to the quotient $Q^{2}$ of $P^{2}$ where two adjacent edges are identified. If $Z_{0}$ does not coincide with $S_{0}$ then by Lemma 27 we may find a 2-simplex $\sigma \subset Z_{0}-S_{0}$ with $\partial \sigma$ null-homotopic in $Z-\operatorname{Int}(\sigma)$. Removing $\sigma$ from $S$ does not change the fundamental group and leads to a subcomplex of $S$ contradicting the minimality property (b). Hence $Z_{0}=S_{0}$.

This shows that $S$ cannot have minimal cycles of type A and any minimal cycle of type B contained in $S$ must have $S_{0}$ as its core.

Let $Z \subset S$ be a minimal cycle of type B contained in $S$. Then $S_{0} \subset Z$ and by Lemma $27 Z$ is simply connected and therefore the inclusion $S_{0} \subset Z$ induces the trivial homomorphism on the fundamental groups. Hence by minimality (b) we have $Z=S$. This shows that $b_{2}(S) \leq 1$. In particular,

$$
\chi\left(S, S_{0}\right)=\chi(S)-\chi\left(S_{0}\right) \leq 1 .
$$

Next we show that $L\left(S, S_{0}\right)=L(S)-L\left(S_{0}\right) \leq-3$. Let $S^{\prime}=\overline{S-S_{0}}$ be the closure of the complement of $S_{0}$ in $S$. The intersection $S_{0} \cap S^{\prime}$ is a graph $\Gamma$. If $b_{1}(\Gamma)=0$ then the inclusion $S_{0} \rightarrow S$ is injective on the fundamental groups. Hence $b_{1}(\Gamma) \geq 1$ and thus $f_{1}(\Gamma) \geq 3$ implying that

$$
L\left(S, S_{0}\right)=L(S)-L\left(S_{0}\right) \leq-3 .
$$

Recall that for any simplicial complex $X$ of dimension $\leq 2$ one has

$$
\begin{aligned}
& 3 \chi(X)+L(X)=3 f_{0}(X)-f_{1}(X), \\
& 2 \chi(X)+L(X)=2 f_{0}(X)-f_{2}(X),
\end{aligned}
$$

see (34). Using these equalities and the above relations we obtain

$$
\begin{aligned}
& 3 f_{0}\left(S, S_{0}\right)-f_{1}\left(S, S_{0}\right)=3 \chi\left(S, S_{0}\right)+L\left(S, S_{0}\right) \leq 0, \\
& 2 f_{0}\left(S, S_{0}\right)-f_{2}\left(S, S_{0}\right)=2 \chi\left(S, S_{0}\right)+L\left(S, S_{0}\right) \leq-1 .
\end{aligned}
$$

We conclude that for any $S \in \mathcal{L}_{\epsilon}$ one has

$$
\begin{aligned}
& f_{1}\left(S, S_{0}\right) \geq 3 f_{0}\left(S, S_{0}\right), \\
& f_{2}\left(S, S_{0}\right) \geq 2 f_{0}\left(S, S_{0}\right)+1 .
\end{aligned}
$$

Now we see that

$$
n^{f_{0}\left(S, S_{0}\right)} \prod_{i=0}^{2} p_{i}^{f_{i}\left(S, S_{0}\right)} \leq\left[n p_{0} p_{1}^{3} p_{2}^{2}\right]^{f_{0}\left(S, S_{0}\right)} \cdot p_{2} \rightarrow 0 .
$$

Applying Theorem 4 we obtain that with probability tending to one, $Y \in \Omega_{n}^{r}$ admits an embedding of $S_{0}$ which does not extend to an embedding $S \rightarrow Y$ for every $S \in \mathcal{L}_{\epsilon}$. 
Let $c_{\epsilon}>0$ be the constant given by Theorem 5 . Consider the set $\Omega_{n}^{\prime} \subset \Omega_{n}^{r}$ consisting of complexes $Y \in \Omega_{n}^{r}$ satisfying the following three conditions:

(1) $Y$ satisfies $I(Y) \geq c_{\epsilon}$;

(2) any pure 2-dimensional subcomplex $S \subset Y$ with

$$
f_{2}(S) \leq 3 \cdot c_{\epsilon}^{-1}+10
$$

is $\epsilon$-admissible.

(3) $Y$ contains a copy of $S_{0}$ as a subcomplex such that there exists no complex $S \in \mathcal{L}_{\epsilon}$ for which the embedding $S_{0} \rightarrow Y$ can be extended to an embedding $S \rightarrow Y$.

By Theorem 5, Lemma 14, Theorem 1 and Theorem 4.4 from [12], the probability that $Y$ belongs to $\Omega_{n}^{\prime}$ tends to one as $n \rightarrow \infty$.

Let us explain that for $Y \in \Omega_{n}^{\prime}$ the embedding $S_{0} \rightarrow Y$ is essential. For any embedding $S_{0} \subset Y$ which is not essential there exists a simplicial disc $D$ with at most $3 \cdot c_{\epsilon}^{-1} 2$-simplexes and $\partial D=C$ where $C \subset S_{0}$ is the non-null-homotopic loop on $S_{0}$ of length 3 . The disc $D$ is simplicially mapped into $Y$ extending the embedding $C \rightarrow Y$ (this follows from the inequality $I(Y) \geq c_{\epsilon}^{-1}$ given by Theorem 5 ). The union of $S_{0}$ and the image of this disc is a subcomplex $S \subset Y$ containing $S_{0}$ which satisfies the properties (a) and (c). Property (d) is satisfied because of Lemma 14 . If the minimality property (b) is not satisfied, then instead of $S$ we can take an appropriate smaller subcomplex. Hence any non-essential embedding $C \rightarrow Y$ can be extended to an embedding $S \rightarrow Y$ for some $S \in \mathcal{L}_{\epsilon}$.

This completes the proof.

Note that the assumption $p_{2} \rightarrow 0$ was essentially used in the proof since in the product $(41)$ the exponent $f_{0}\left(S, S_{0}\right)$ may happen to be 0 . We believe that Theorem 34 will remain true under a weaker assumption $p_{2}<c<1$ where $c$ is a constant.

Next we state another result assuming that $p_{2}=1$, which is a generalization of Theorem 7.2 from [11].

Theorem 37. Let $Y \in \Omega_{n}^{r}$ be a random simplicial complex with respect to the probability measure $\mathbb{P}_{r, \mathfrak{p}}$ where $\mathfrak{p}=\left(p_{0}, p_{1}, \ldots, p_{r}\right)$. Assume that

$$
\begin{aligned}
p_{2} & =1, \\
n p_{0} p_{1}^{30 / 11} & \rightarrow \infty,
\end{aligned}
$$

and for some $\epsilon>0$,

$$
\left(n p_{0}\right)^{1+\epsilon} p_{1}^{3} \rightarrow 0
$$

Then the fundamental group $\pi_{1}(Y)$ has nontrivial elements of order two, a.a.s. 
Remark 38. In the special case when

$$
p_{i}=n^{-\alpha_{i}}
$$

with $\alpha_{i} \geq 0$ constant, where $i=0,1, \ldots$ Theorem 37 states that the fundamental group of a random complex $Y$ has a nontrivial element of order 2 assuming that

$$
\begin{aligned}
\alpha_{0}+\frac{30}{11} \alpha_{1} & <1, \\
\alpha_{0}+3 \alpha_{1} & >1, \\
\alpha_{2} & =0 .
\end{aligned}
$$

This result was proven in [11] in the special case when $\alpha_{0}=0$.

Remark 39. The main distinctions between Theorems 34 and 37 are the assumptions regarding the behavior of $p_{2}$. The 2-torsion in the fundamental group is generated by essential embeddings of the real projective plane $P^{2}$. In the case of Theorem 34 we are dealing with the embeddings of the minimal triangulation $S_{0}$ of $P^{2}$. However, in the case when $p_{2}=1$ the triangular essential loop of $S_{0}$ bounds a triangle in $Y$. This explains that in the case $p_{2}=1$ one has consider clean triangulations of $P^{2}$. Recall that a triangulation of a 2-complex is called clean if for any clique of 3 vertices $\left\{v_{0}, v_{1}, v_{2}\right\}$ the complex contains also the 2 -simplex $\left(v_{0}, v_{1}, v_{2}\right)$.

We shall use the following fact: any clean triangulation of the projective plane $P^{2}$ contains at least 11 vertices and 30 edges, see [17]. The minimal clean triangulation is shown in Fig. 5; the antipodal points of the circle must be identified.

Proof of Theorem 37. The proof is very similar to the proof of Theorem 34; we shall indicate the main steps and emphasize the main distinctions.

Let $S_{0}$ be the minimal clean triangulation of the real projective plane shown in Fig. 5. It is balanced (by Theorem 4.4 from [12]) and as in the proof of Theorem 34

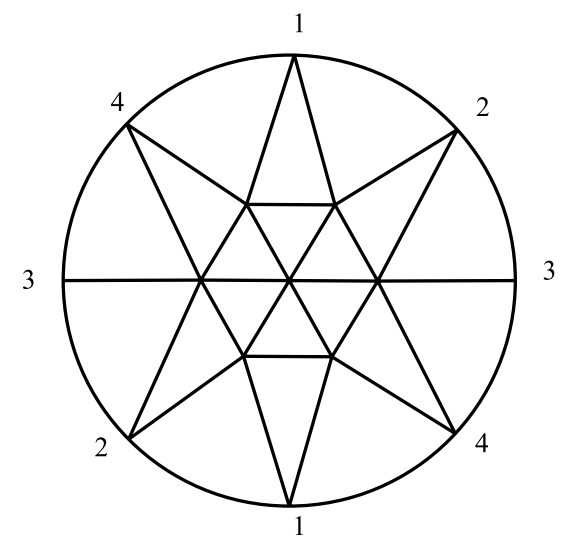

Fig. 5. The minimal clean triangulation of $P^{2}$, according to [17]. 
we find that for $n p_{0} p_{1}^{30 / 11} p_{2}^{20 / 11} \rightarrow \infty$ the complex $S_{0}$ simplicially embeds into $Y$, a.a.s. Since we assume that $p_{2}=1$, this condition coincides with (43).

As above, we want to show that $Y$ contains $S_{0}$ as "an essential subcomplex".

Consider the set $\mathcal{L}_{\epsilon}$ of isomorphism types of pairs $\left(S, S_{0}\right)$ where $S$ is a finite 2-complex containing $S_{0}$ satisfying the following conditions:

(a) the inclusion $S_{0} \rightarrow S$ induces the trivial homomorphism of the fundamental groups;

(b) $S$ is minimal in the sense that for any proper subcomplex $S_{0} \subset S^{\prime} \subsetneq S$ the inclusion $S_{0} \rightarrow S^{\prime}$ induces an injective homomorphism $\pi_{1}\left(S_{0}\right) \rightarrow \pi_{1}\left(S^{\prime}\right)$;

(c) $f_{2}(S) \leq 4 \cdot c_{\epsilon}^{-1}+20$;

(d) $S$ is $\epsilon$-admissible.

As in the proof of Theorem 34 one obtains that each $S \in \mathcal{L}_{\epsilon}$ is pure and

$$
\chi\left(S, S_{0}\right)=\chi(S)-\chi\left(S_{0}\right) \leq 1 .
$$

Let us show that

$$
L\left(S, S_{0}\right)=L(S)-L\left(S_{0}\right) \leq-4
$$

(unlike the case of Theorem 34). Let $S^{\prime}=\overline{S-S_{0}}$ be the closure of the complement of $S_{0}$ in $S$. The intersection $S_{0} \cap S^{\prime}$ is a graph $\Gamma$. If $b_{1}(\Gamma)=0$ then the inclusion $S_{0} \rightarrow S$ is injective on the fundamental groups. Hence $b_{1}(\Gamma) \geq 1$. Thus $\Gamma$ has a cycle and has at least 3 edges. However if $f_{1}(\Gamma)=3$ then the cycle of $\Gamma$ bounds a 2-simplex on $S_{0}$ and hence the inclusion $S_{0} \rightarrow S$ is injective on the fundamental group, in contradiction with our assumption (a). Thus, $f_{1}(\Gamma) \geq 4$ implying (46).

Combining (45) and (46) with (40), we conclude that for any $S \in \mathcal{L}_{\epsilon}$ one has

$$
\begin{aligned}
& f_{1}\left(S, S_{0}\right) \geq 3 f_{0}\left(S, S_{0}\right)+1, \\
& f_{2}\left(S, S_{0}\right) \geq 2 f_{0}\left(S, S_{0}\right)+2,
\end{aligned}
$$

and we see that

$$
\begin{aligned}
n^{f_{0}\left(S, S_{0}\right)} \prod_{i=0}^{2} p_{i}^{f_{i}\left(S, S_{0}\right)} & \leq\left[n p_{0} p_{1}^{3} p_{2}^{2}\right]^{f_{0}\left(S, S_{0}\right)} \cdot p_{1} p_{2}^{2} \\
& =\left[n p_{0} p_{1}^{3}\right]^{f_{0}\left(S, S_{0}\right)} \cdot p_{1} \\
& \leq p_{1} \rightarrow 0 .
\end{aligned}
$$

Note that $p_{1} \rightarrow 0$ as follows from (44).

The rest of the proof is identical to the proof of Theorem 34 .

This completes the proof.

\section{Higher Torsion}

In this section we show that random simplicial complexes have no odd torsion for a large range of probability parameters. 
Theorem 40. Let $m \geq 3$ be a fixed odd prime. Consider a random simplicial complex $Y \in \Omega_{n}^{r}, r \geq 2$, with respect to the probability measure $\mathbb{P}_{r, \mathfrak{p}}$ where $\mathfrak{p}=$ $\left(p_{0}, p_{1}, \ldots, p_{r}\right)$. Assume that $n p_{0} \rightarrow \infty$ and for some $\epsilon>0$ one has

$$
\left(n p_{0}\right)^{1+\epsilon} p_{1}^{3} p_{2}^{2} \rightarrow 0 .
$$

Then a random complex $Y \in \Omega_{n}^{r}$ with probability tending to 1 has the following property: the fundamental group of any connected subcomplex $Y^{\prime} \subset Y$ has no $m$ torsion.

Remark 41. In the special case when

$$
p_{i}=n^{-\alpha_{i}}
$$

with $\alpha_{i} \geq 0$ being constant, where $i=0,1, \ldots$ Theorem 40 states that for any odd prime $m \geq 3$ the fundamental group of a random complex $Y$ has no nontrivial elements of order $m$ assuming that

$$
\alpha_{0}<1 \quad \text { and } \quad \alpha_{0}+3 \alpha_{1}+2 \alpha_{2}>1 \text {. }
$$

This result was proven in [10] in the special case when $\alpha_{0}=\alpha_{1}=0$ and in [11] in the special case $\alpha_{0}=\alpha_{2}=0$.

The proof of Theorem 40 given below uses an auxiliary material which we now describe.

Let $f_{m}: S^{1} \rightarrow S^{1}$ denote the canonical degree $m$ map, $f_{m}(z)=z^{m}$ where $z \in S^{1}$; we think of $S^{1}$ as being the unit circle on the complex plane. Any simplicial complex $\Sigma$ homeomorphic

$$
M\left(\mathbb{Z}_{m}, 1\right)=S^{1} \cup_{f_{m}} e^{2}
$$

is called a Moore surface.

Everywhere in this section we shall assume that $m \geq 3$ is a fixed odd prime.

Then any Moore surface $\Sigma$ has a well-defined circle $C \subset \Sigma$ (called the singular circle) which is the union of all edges of degree $m$; all other edges of $\Sigma$ have degree 2 . Clearly, the homotopy class of the singular circle generates the fundamental group $\pi_{1}(\Sigma) \simeq \mathbb{Z}_{m}$

Define an integer $N_{m}(Y) \geq 0$ associated to any connected simplicial complex $Y \in \Omega_{n}^{r}$. If $\pi_{1}(Y)$ has no $m$-torsion we set $N_{m}(Y)=0$. If $\pi_{1}(Y)$ has elements of order $m$ we consider homotopically nontrivial simplicial maps $\gamma: C_{r} \rightarrow Y$, where $C_{r}$ is the simplicial circle with $r$ edges, such that

(a) $\gamma^{m}$ is null-homotopic (as a free loop in $Y$ );

(b) $r$ is minimal: for $r^{\prime}<r$ any simplicial loop $\gamma: C_{r^{\prime}} \rightarrow Y$ satisfying (a) is homotopically trivial.

Any such simplicial map $\gamma: C_{r} \rightarrow Y$ can be extended to a simplicial map $f: \Sigma \rightarrow Y$ of a Moore surface $\Sigma$ such that the singular circle $C$ of $\Sigma$ is isomorphic to $C_{r}$ and $f \mid C=\gamma$. We shall say that a simplicial map $f: \Sigma \rightarrow Y$ is m-minimal if it satisfies 
(a), (b) and the number of 2-simplexes in $\Sigma$ is the smallest possible. Clearly, any $m$-minimal map $\Sigma \rightarrow Y$ induces an injective homomorphism $\pi_{1}(\Sigma) \simeq \mathbb{Z}_{m} \rightarrow \pi_{1}(Y)$. We denote by

$$
N_{m}(Y) \in \mathbb{Z}
$$

the number of 2-simplexes in a triangulation of a Moore surface $\Sigma$ admitting an $m$-minimal map $f: \Sigma \rightarrow Y$.

We recall Lemma 4.7 from [10]:

Lemma 42. Let $Y$ be a simplicial complex satisfying $I(Y) \geq c>0$ and let $m \geq 3$ be an odd prime. Then one has

$$
N_{m}(Y) \leq\left(\frac{6 m}{c}\right)^{2} .
$$

Using this lemma we may obtain a global upper bound on the numbers $N_{m}(Y)$ for random complexes:

Theorem 43. Assume that the probability multi-parameter $\mathfrak{p}=\left(p_{0}, p_{1}, \ldots, p_{r}\right)$, where $r \geq 2$, satisfies

$$
n p_{0} \rightarrow \infty, \quad\left(n p_{0}\right)^{1+\epsilon} p_{1}^{3} p_{2}^{2} \rightarrow 0
$$

with $\epsilon>0$ is fixed. Let $m \geq 3$ be an odd prime. Then there exists a constant $C_{\epsilon}>0$ such that a random complex $Y \in \Omega_{n}^{r}$ with probability tending to 1 has the following property: for any subcomplex $Y^{\prime} \subset Y$ one has

$$
N_{m}\left(Y^{\prime}\right) \leq C_{\epsilon}
$$

Proof. We know from Theorem 5 that, with probability tending to 1 , a random complex $Y$ has the following property: for any subcomplex $Y^{\prime} \subset Y$ one has $I\left(Y^{\prime}\right) \geq$ $c_{\epsilon}>0$ where $c_{\epsilon}>0$ is the constant given by Theorem 5 . Then, setting $C_{\epsilon}=\left(\frac{6 m}{c_{\epsilon}}\right)^{2}$, the inequality (50) follows from Lemma 42.

Now we are ready to present the proof of Theorem 40:

Proof of Theorem 40. Let $c_{\epsilon}>0$ be the number given by Theorem 5 . Consider the finite set of all isomorphism types of Moore surfaces $\mathcal{S}_{m}=\{\Sigma\}$ having at most $\left(\frac{6 m}{c_{\epsilon}}\right)^{2}$ 2-dimensional simplexes. Let $\mathcal{X}_{m}$ denote the set of isomorphism types of images of all surjective simplicial maps $\Sigma \rightarrow X$ inducing injective homomorphisms $\pi_{1}(\Sigma)=\mathbb{Z}_{m} \rightarrow \pi_{1}(X)$, where $\Sigma \in \mathcal{S}_{m}$. The set $\mathcal{X}_{m}$ is also finite.

From Theorem 43 we obtain that, with probability tending to one, for any subcomplex $Y^{\prime} \subset Y$, either $\pi_{1}\left(Y^{\prime}\right)$ has no $m$-torsion, or there exists an $m$-minimal map $f: \Sigma \rightarrow Y^{\prime}$ where $\Sigma$ is a Moore surface having at most $\left(\frac{6 m}{c_{\epsilon}}\right)^{2} 2$-simplexes of dimension 2; in the second case the image $X=f(\Sigma)$ is a subcomplex of $Y^{\prime}$ and $f: \Sigma \rightarrow X$ induces a monomorphism $\pi_{1}(\Sigma) \rightarrow \pi_{1}(X)$, i.e. $X \in \mathcal{X}_{m}$. Denote by 
$\mathcal{X}_{m}^{\prime} \subset \mathcal{X}_{m}$ the set of complexes $X \in \mathcal{X}_{m}$ such that their 2-dimensional pure parts are $\epsilon$-admissible. We may apply Lemma 14 (using the upper bound $f_{2}(X) \leq C=$ $\left.\left(\frac{6 m}{c_{\epsilon}}\right)^{2}\right)$ to conclude that the pure part of the image $X=f(\Sigma)$ of any $m$-minimal map $f: \Sigma \rightarrow Y$ belongs to $\mathcal{X}_{m}^{\prime}$. However, by Theorem 28 the fundamental group of any $X \in \mathcal{X}_{m}^{\prime}$ is a free product of several copies of $\mathbb{Z}$ and $\mathbb{Z}_{2}$ and hence it has no $m$-torsion. This leads to a contradiction which shows that the fundamental group of any subcomplex $Y^{\prime} \subset Y$ does not have $m$-torsion, a.a.s.

\section{Asphericity and the Whitehead Conjecture}

Recall that a connected simplicial complex $Y$ is said to be aspherical if $\pi_{k}(Y)=0$ for all $k \geq 2$. A 2-dimensional connected simplicial complex $Y$ is aspherical if and only if $\pi_{2}(Y)=0$. The well-known Whitehead Conjecture states that any subcomplex of an aspherical 2-complex is also aspherical, see $[1,5,6,24]$. At the time of writing the Whitehead Conjecture is still open.

In this section we show that for random simplicial 2-complexes, with probability tending to one, the asphericity of a subcomplex of a random complex is equivalent to the absence of "small bubbles". It follows that in the random setting any subcomplex of an aspherical 2-complex is also aspherical, supporting a probabilistic analogue of the Whitehead Conjecture. See also [8, 10].

Theorem 44. Consider a random simplicial complex $Y \in \Omega_{n}^{r}, r \geq 2$, with respect to the probability measure $\mathbb{P}_{r, \mathfrak{p}}$ where $\mathfrak{p}=\left(p_{0}, p_{1}, \ldots, p_{r}\right)$. Assume that $n p_{0} \rightarrow \infty$ and for some $\epsilon>0$ one has

$$
\left(n p_{0}\right)^{1+\epsilon} p_{1}^{3} p_{2}^{2} \rightarrow 0
$$

Let $c_{\epsilon}$ be the constant given by Theorem 5. Then, a random complex $Y \in \Omega_{n}^{r}$ has the following property with probability tending to 1 as $n \rightarrow \infty$ : any subcomplex $Y^{\prime} \subset Y^{(2)}$ is aspherical if and only if every pure subcomplex $S \subset Y^{\prime}$ satisfying

$$
f_{2}(S) \leq C=16^{2} c_{\epsilon}^{-2}
$$

is collapsible to a graph. In particular, under the above assumptions, with probability tending to one, any aspherical subcomplex of $Y^{(2)}$ satisfies the Whitehead Conjecture.

The proof given below in this section will use the following auxiliary material.

Let $Y$ be a simplicial complex with $\pi_{2}(Y) \neq 0$. As in [10] and [11], we define a numerical invariant $M(Y) \in \mathbb{Z}, M(Y) \geq 4$, as the minimal number of faces in a 2-complex $\Sigma$ homeomorphic to the sphere $S^{2}$ such that there exists a homotopically nontrivial simplicial map $\Sigma \rightarrow Y$. 
We define $M(Y)=0$, if $\pi_{2}(Y)=0$.

Lemma 45. (See Corollary 5.4 in [10]) Let $Y$ be a 2-complex with $I(Y) \geq c>0$. Then

$$
M(Y) \leq\left(\frac{16}{c}\right)^{2} .
$$

Combining this lemma with Theorem 5 we obtain:

Lemma 46. Assume that

$$
n p_{0} \rightarrow \infty \quad \text { and } \quad\left(n p_{0}\right)^{1+\epsilon} p_{1}^{3} p_{2}^{2} \rightarrow 0
$$

for some $\epsilon>0$. Then there exists a constant $C_{\epsilon}>0$ such that a random complex $Y \in$ $\Omega_{n}^{r}$ has the following property with probability tending to one: for any subcomplex $Y^{\prime} \subset Y^{(2)}$ one has

$$
M\left(Y^{\prime}\right) \leq C_{\epsilon}
$$

Hence, if we want to find homotopically nontrivial simplicial maps from $S^{2}$ to a random complex, it is sufficient to consider triangulations of $S^{2}$ having at most $C_{\epsilon}$ 2-simplexes.

The following lemma will be used in the proof of Theorem 44 .

Lemma 47. Let $S$ be a connected admissible 2-complex (see Definition 9). Then $S$ is aspherical if and only if $S$ is simplicially collapsible to a graph.

Proof. Let $S$ be a connected admissible 2-complex satisfying $\pi_{2}(S)=0$. Performing all possible simplicial collapses of 2 -simplexes we may find a closed pure subcomplex $S^{\prime} \subset S$ without free edges. We need to show that $S^{\prime}$ is 1-dimensional. We shall assume below that $\operatorname{dim} S^{\prime}=2$ and arrive at a contradiction.

If $b_{2}\left(S^{\prime}\right) \geq 1$ then $S^{\prime}$ contains an admissible minimal cycle $Z \subset S^{\prime}$ as a subcomplex. Using Lemmas 23 and 27 (depending on whether $Z$ is of type A or B) we find a 2-simplex $\sigma \subset Z$ such that $\partial \sigma$ is null-homotopic in $Z-\operatorname{Int}(\sigma)$. Then $S^{\prime}$ is homotopy equivalent to the wedge $\left(S^{\prime}-\operatorname{Int}(\sigma)\right) \vee S^{2}$ contradicting the assumption $\pi_{2}\left(S^{\prime}\right)=0$.

Hence we must assume that $b_{2}\left(S^{\prime}\right)=0$. Using Corollary 18 we see that every strongly connected component of $S^{\prime}$ is homeomorphic to either $P^{2}$ or to the quotient $Q^{2}$ of $P^{2}$ with two adjacent edges identified. In both cases we may apply a theorem of Cockcroft [6] (see also Adams [1]) which claims that $\pi_{2}\left(S^{\prime}\right) \neq 0$, in contradiction with our hypothesis.

Lemma 48. Let $S$ be a connected admissible 2-complex. If $S$ is not aspherical then $S$ contains a subcomplex which is homotopy equivalent to one of $S^{2}, S^{2} \vee S^{1}$ or $P^{2}$. 
Proof. Let $S$ be a connected admissible 2-complex; without loss of generality we may assume that $S$ is closed and pure. If $b_{2}(S) \neq 0$ then $S$ contains an admissible minimal cycle $Z \subset S$ and $Z$ is homotopy equivalent to either $S^{2}, S^{2} \vee S^{1}$ (in the case of type A, see Lemma 23), or to $P^{2}$ (in the case of type B, see Lemma 27). In the case $b_{2}(S)=0$ we invoke Corollary 18 .

Proof of Theorem 44. Consider the set $\mathcal{S}$ of isomorphism types of all pure 2complexes $S$ satisfying $f_{2}(S) \leq C$ where $C$ is defined in the statement of Theorem 44. We may represent $\mathcal{S}$ as the disjoint union $\mathcal{S}=\mathcal{S}^{\prime} \cup \mathcal{S}^{\prime \prime}$ where the complexes $S \in \mathcal{S}^{\prime}$ are $\epsilon$-admissible and the complexes $S \in \mathcal{S}^{\prime \prime}$ are not.

Let $\Omega_{n}^{\prime} \subset \Omega_{n}^{r}$ be the set of complexes $Y \in \Omega_{n}^{r}$ such that (a) no $S \in \mathcal{S}^{\prime \prime}$ can be embedded into $Y$ and (b) each $Y \in \Omega_{n}^{\prime}$ satisfies the conclusion of Lemma 46. By Lemmas 14 and 46 we know that $\mathbb{P}_{r, \mathfrak{p}}\left(\Omega_{n}^{\prime}\right) \rightarrow 1$ as $n \rightarrow \infty$.

Suppose that $Y \in \Omega_{n}^{\prime}$ and let $Y^{\prime} \subset Y^{(2)}$ be an aspherical subcomplex. For any pure subcomplex $S \subset Y^{\prime}$ with $f_{2}(S) \leq C$ we know that $S \in \mathcal{S}^{\prime}$ and by Lemma 48 either $S$ is aspherical or it contains a subcomplex homotopy equivalent to either $S^{2}$ or $P^{2}$. Both these possibilities would imply $\pi_{2}\left(Y^{\prime}\right) \neq 0$ (the case of $S^{2}$ is obvious and the case of $P^{2}$ follows from the work of Cockcroft [6], see also [1]). Thus we see that in $Y^{\prime} \subset Y$ is aspherical then any subcomplex $S \subset Y^{\prime}$ with $f_{2}(S)<C$ is also aspherical; the latter due to Lemma 47 is equivalent for $S$ to be collapsible to a graph.

We now prove the inverse implication by assuming that $Y^{\prime} \subset Y$ is non-aspherical and $Y \in \Omega_{n}^{\prime}$. By Lemma 46 there exists a 2-complex $\Sigma$ homeomorphic to a 2sphere with $f_{2}(\Sigma) \leq C=16^{2} c_{\epsilon}^{-2}$ and a homotopically nontrivial simplicial map $\phi: \Sigma \rightarrow Y^{\prime}$. We denote $S=\phi(\Sigma)$ and thus we have $f_{2}(S) \leq f_{2}(\Sigma) \leq C$ and $\pi_{2}(S) \neq 0$. Hence we conclude that if $Y^{\prime} \subset Y$ is not aspherical then there exists a subcomplex $S \subset Y^{\prime}, S \in \mathcal{S}^{\prime}$, and $f_{2}(S) \leq C$ and $\pi_{2}(S) \neq 0$.

This completes the proof.

\section{Geometric and Cohomological Dimension of the Fundamental Group of a Random Simplicial Complex}

Theorem 49. Consider a random simplicial complex $Y \in \Omega_{n}^{r}$ with respect to the multi-parameter probability measure $\mathbb{P}_{r, \mathfrak{p}}$, where $\mathfrak{p}=\left(p_{0}, p_{1}, \ldots, p_{r}\right), r \geq 2$. Assume that $n p_{0} \rightarrow \infty$ and

$$
n p_{0} p_{1}^{5 / 2} p_{2}^{5 / 3} \rightarrow 0
$$

Then for any choice of the base point $y_{0} \in Y$ the fundamental group $\pi_{1}\left(Y, y_{0}\right)$ has geometric dimension at most 2 , a.a.s. In particular, the group $\pi_{1}\left(Y, y_{0}\right)$ has cohomological dimension at most 2 and is torsion free, a.a.s.

Proof. We will show that with probability tending to one a random complex $Y \in$ $\Omega_{n}^{r}$ contains a 2-dimension subcomplex $Y^{\prime} \subset Y$ such that

(1) $Y^{(1)}=Y^{\prime(1)}$; 
(2) $\pi_{1}\left(Y^{\prime}, y_{0}\right) \simeq \pi_{1}\left(Y, y_{0}\right)$ for any vertex $y_{0} \in Y$;

(3) any connected component of $Y^{\prime}$ is aspherical.

This would clearly imply the statement of Theorem 49.

Note that our assumption (51) implies the condition (11) for any $\epsilon<1 / 5$. Hence we may apply Theorems 5 and 44 . To be specific we may set $\epsilon=1 / 10$ and denote by $c_{\epsilon}>0$ the constant given by Theorem 5 .

Denote by $\Omega_{n}^{\prime} \subset \Omega_{n}^{r}$ the set of complexes $Y \in \Omega_{n}^{r}$ satisfying the following conditions:

(a) For $Y \in \Omega_{n}^{\prime}$ any 2-dimensional pure subcomplex $S \subset Y$ with $f_{2}(S) \leq C$ is admissible. Here $C$ denotes $16^{2} c_{\epsilon}^{-2}$.

(b) For $Y \in \Omega_{n}^{\prime}$ a subcomplex $Y^{\prime} \subset Y^{(2)}$ is aspherical if and only if every subcomplex $S \subset Y^{\prime}$ satisfying $f_{2}(S)<C$ is collapsible to a graph.

(c) Any complex $Y \in \Omega_{n}^{\prime}$ has no closed admissible 2-dimensional pure subcomplexes $S \subset Y$ with $f_{2}(S) \leq C$ satisfying $b_{2}(S)=0$.

We know that $\mathbb{P}_{r, \mathfrak{p}}\left(\Omega_{n}^{\prime}\right) \rightarrow 1$ due to Lemma 14 and Theorem 44; to explain that the property (c) can be achieved we observe that by Corollary 18 any such $S$ is either a triangulation of $P^{2}$ or the quotient $Q^{2}$ of $P^{2}$ obtained by identifying two adjacent edges in certain triangulation. Assuming that $S$ is a triangulation of $P^{2}$ we have $f_{0}(S)-f_{1}(S)+f_{2}(S)=1,3 f_{2}(S)=2 f_{1}(S)$ and $f_{0}(S) \geq 6$ implying that $f_{1}(S) \geq 15$ and $f_{2}(S) \geq 10$ and also

$$
\begin{aligned}
& \frac{f_{0}(S)}{f_{1}(S)}=\frac{1}{3}+\frac{1}{f_{1}(S)} \leq 2 / 5, \\
& \frac{f_{0}(S)}{f_{2}(S)}=\frac{1}{2}+\frac{1}{f_{2}(S)} \leq 3 / 5 .
\end{aligned}
$$

Therefore,

$$
n p_{0} p_{1}^{\frac{f_{1}(S)}{f_{0}(S)}} p_{2}^{\frac{f_{2}(S)}{f_{0}(S)}} \leq n p_{0} p_{1}^{5 / 2} p_{2}^{5 / 3} \rightarrow 0
$$

because of our assumption (51). In the case when $S$ is obtained from a triangulation $T$ of $P^{2}$ by identifying two adjacent edges we have $f_{0}(S)=f_{0}(T)-1, f_{1}(S)=$ $f_{1}(T)-1$ and $f_{2}(S)=f_{2}(T)$. Then

$$
\frac{f_{i}(T)}{f_{0}(T)} \geq \frac{f_{i}(S)}{f_{0}(S)}, \quad i=1,2,
$$

and we obtain

$$
n p_{0} p_{1}^{\frac{f_{1}(T)}{f_{0}(T)}} p_{2}^{\frac{f_{2}(T)}{f_{0}(T)}} \leq n p_{0} p_{1}^{\frac{f_{1}(S)}{f_{0}(S)}} p_{2}^{\frac{f_{2}(S)}{f_{0}(S)}} \rightarrow 0
$$

as shown above. Hence our statement follows by invoking Theorem 1 .

Given a complex $Y \in \Omega_{n}^{\prime}$, consider an admissible minimal cycle $Z \subset Y$ with $f_{2}(Z) \leq C$. Using Lemmas 23 and 27 we may find a 2-simplex $\sigma \subset Z \subset Y$ such that 
removing it we do not change the fundamental group. Therefore we may inductively obtain a sequence of subcomplexes

$$
Y_{0}=Y^{(2)} \supset Y_{1} \supset Y_{2} \cdots \supset Y_{N}
$$

with each complex $Y_{i+1}$ obtained from the previous $Y_{i}$ by removing the interior of a 2-simplex $\sigma \subset Y_{i}$ such that $\partial \sigma$ is null-homotopic in $Y_{i}-\operatorname{Int}(\sigma)$. Let $Y^{\prime}=Y_{N} \subset Y$ be the final complex in this sequence.

We claim that the obtained complex $Y^{\prime}$ is aspherical. Let $S \subset Y^{\prime}$ be a pure subcomplex with $f_{2}(S) \leq C$. Then $b_{2}(S)=0$ since otherwise $Y^{\prime}$ would contain an admissible minimal cycle $Z$ with $f_{2}(Z) \leq C$ contradicting our construction (here we use (a)). By (c) the complex $Y^{\prime}$ contains no closed admissible 2-dimensional pure subcomplexes $S \subset Y$ with $f_{2}(S) \leq C$ satisfying $b_{2}(S)=0$. Hence any subcomplex $S \subset Y^{\prime}$ with $f_{2}(S) \leq C$ is collapsible to a graph. By property (b) the complex $Y^{\prime}$ is aspherical.

This completes the proof.

Next we state an analogue of Theorem 49 in the special case when $p_{2}=1$.

Theorem 50. Consider a random simplicial complex $Y \in \Omega_{n}^{r}$ with respect to the multi-parameter probability measure $\mathbb{P}_{r, \mathfrak{p}}$, where $\mathfrak{p}=\left(p_{0}, p_{1}, \ldots, p_{r}\right)$. Assume that $n p_{0} \rightarrow \infty, r \geq 2$ and $p_{2}=1$ and besides,

$$
n p_{0} p_{1}^{30 / 11} \rightarrow 0
$$

Then for any choice of the base point $y_{0} \in Y$ the fundamental group $\pi_{1}\left(Y, y_{0}\right)$ has geometric dimension at most 2 , a.a.s. In particular, the group $\pi_{1}\left(Y, y_{0}\right)$ has cohomological dimension at most 2 and is torsion free, a.a.s.

Proof. The proof is similar to the proof of Theorem 49. The only difference is that in the case when $p_{2}=1$ we have a different requirement (52) on the absence of embeddings of clean triangulations of the real projective plane $P^{2}$ into a random 2-complex.

Theorems 49 and 50 complement Theorems 34 and 37 about the existence of 2 -torsion.

Remark 51. In the special case when $p_{i}=n^{-\alpha_{i}}$ with $\alpha_{i} \geq 0$ constants, where $i=0,1, \ldots$ Theorem 49 states that the fundamental group of a random complex $Y$ has geometric dimension at most 2 assuming that $\alpha_{0}<1$ and either

$$
\alpha_{0}+\frac{5}{2} \alpha_{1}+\frac{5}{3} \alpha_{2}>1
$$

or

$$
\alpha_{0}+\frac{30}{11} \alpha_{1}>1 \quad \text { and } \quad \alpha_{2}=0
$$




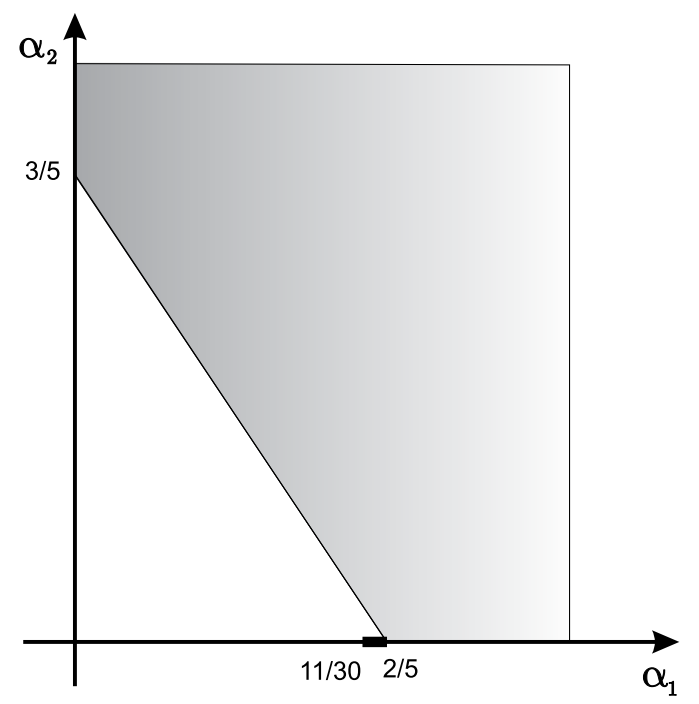

Fig. 6. The region on the plane of $\alpha_{1}, \alpha_{2}$ where the geometric dimension of the fundamental group $\pi_{1}(Y)$ equals 2 .

See Fig. 6; the corresponding set is represented by the shaded area together with an open interval on the $\alpha_{1}$ axis.

These results were proven in [10] in the special case when $\alpha_{0}=\alpha_{1}=0$ and in [11] in the case when $\alpha_{0}=\alpha_{2}=0$. The results of this section shed light on the probabilistic version of the Eilenberg-Ganea Conjecture, see [4, 15].

\section{Appendix: Proof of Theorem 15}

Definition 52. (See [10]) We say that a finite 2-complex $X$ is tight if for any proper subcomplex $X^{\prime} \subset X, X^{\prime} \neq X$, one has $I\left(X^{\prime}\right)>I(X)$.

Clearly, one has

$$
I(X) \geq \min \{I(Y) ; Y \subset X \text { is a tight subcomplex }\} .
$$

By (53) it is obvious that it is enough to prove Theorem 15 under the additional assumption that $X$ is tight.

Remark 53. Suppose that $X$ is pure and tight and suppose that $\gamma: S^{1} \rightarrow X$ is a simplicial loop with the ratio $|\gamma| \cdot A_{X}(\gamma)^{-1}$ less than the minimum of the numbers $I\left(X^{\prime}\right)$ where $X^{\prime} \subset X$ is a proper subcomplex. Let $b: D^{2} \rightarrow X$ be a minimal spanning disc for $\gamma$; then $b\left(D^{2}\right)=X$, i.e. $b$ is surjective. Indeed, if the image of $b$ does not contain a 2-simplex $\sigma$ then removing it we obtain a subcomplex $X^{\prime} \subset X$ with $A_{X^{\prime}}(\gamma)=A_{X}(\gamma)$ and hence $I\left(X^{\prime}\right) \leq I(X) \leq|\gamma| \cdot A_{X}(\gamma)^{-1}$ contradicting the assumption on $\gamma$.

Lemma 54. If $X$ is an admissible tight complex then $b_{2}(X)=0$. 
Proof. Assume that $b_{2}(X) \neq 0$. Then there exists a admissible minimal cycle $Z \subset X$. Hence, by Lemmas 23 and 27 we may find a 2-simplex $\sigma \subset Z \subset X$ such that $\partial \sigma$ is null-homotopic in $Z-\sigma \subset X-\sigma=X^{\prime}$. Note that $X^{\prime(1)}=X^{(1)}$ and a simplicial curve $\gamma: S^{1} \rightarrow X^{\prime}$ is null-homotopic in $X^{\prime}$ if and only if it is null-homotopic in $X$. Besides, $A_{X}(\gamma) \leq A_{X^{\prime}}(\gamma)$ and hence

$$
\frac{|\gamma|}{A_{X}(\gamma)} \geq \frac{|\gamma|}{A_{X^{\prime}}(\gamma)},
$$

which implies that $I(X) \geq I\left(X^{\prime}\right)$. We obtained a contradiction since $X$ is tight.

Lemma 55. Given $\epsilon>0$ there exists a constant $C_{\epsilon}^{\prime}>0$ such that for every finite pure tight connected $\epsilon$-admissible complex $X$ satisfying $L(X) \leq 0$ one has $I(X) \geq C_{\epsilon}^{\prime}$.

This lemma is similar to Theorem 15 but it has an additional assumption that $L(X) \leq 0$. The assumption $L(X) \leq 0$ can be replaced, without altering the proof, by any assumption of the type $L(X) \leq 1000$, i.e. by any specific upper bound.

Proof of Lemma 55. We show that the number of isomorphism types of complexes $X$ satisfying the conditions of the lemma is finite; hence the proof follows by setting $C_{\epsilon}^{\prime}=\min I(X)$ and using Theorem 28 which gives $I(X)>0$ (since $\pi_{1}(X)$ is hyperbolic) and hence $C_{\epsilon}^{\prime}>0$.

By Lemma 16 we obtain

$$
\mu_{1}\left(X^{\prime}\right) \geq \frac{1+\epsilon}{3} \quad \text { or } \quad \mu_{2}\left(X^{\prime}\right) \geq \frac{1+\epsilon}{2} .
$$

The inequality

$$
\mu_{1}(X)=\frac{1}{3}+\frac{3 \chi(X)+L(X)}{3 f_{1}(X)} \geq \frac{1+\epsilon}{3}
$$

is equivalent to

$$
f_{1}(X) \leq \epsilon^{-1} \cdot(3 \chi(X)+L(X)),
$$

where $f_{1}(X)$ denotes the number of 1-simplexes in $X$. By Lemma 54 we have $\chi(X)=1-b_{1}(X) \leq 1$ and using the assumption $L(X) \leq 0$ we obtain $f_{1}(X) \leq 3 \epsilon^{-1}$. This implies the finiteness of the set of possible isomorphism types of $X$ and the result follows.

The case $\mu_{2}(X) \geq(1+\epsilon) / 2$ is analogous.

We will use a relative isoperimetric constant $I\left(X, X^{\prime}\right) \in \mathbb{R}$ for a pair consisting of a finite 2-complex $X$ and its subcomplex $X^{\prime} \subset X$; it is defined as the infimum of all ratios $|\gamma| \cdot A_{X}(\gamma)^{-1}$ where $\gamma: S^{1} \rightarrow X^{\prime}$ runs over simplicial loops in $X^{\prime}$ which are 
null-homotopic in $X$. Clearly, $I\left(X, X^{\prime}\right) \geq I(X)$ and $I\left(X, X^{\prime}\right)=I(X)$ if $X^{\prime}=X$. Below is a useful strengthening of Lemma 55 .

Lemma 56. Given $\epsilon>0$, let $C_{\epsilon}^{\prime}>0$ be the constant given by Lemma 55. Then for any finite pure, tight, $\epsilon$-admissible and connected 2 -complex $X$ and for a connected subcomplex $X^{\prime} \subset X$ satisfying $L\left(X^{\prime}\right) \leq 0$ one has $I\left(X, X^{\prime}\right) \geq C_{\epsilon}^{\prime}$.

Proof. We show below that under the assumptions on $X, X^{\prime}$ one has

$$
I\left(X, X^{\prime}\right) \geq \min _{Y} I(Y),
$$

where $Y$ runs over all subcomplexes $X^{\prime} \subset Y \subset X$ satisfying $L(Y) \leq 0$. Clearly, any such $Y$ is $\epsilon$-admissible (as a subcomplex of $\epsilon$-admissible complex $X$ ). By Lemma 54 we have that $b_{2}(X)=0$ which implies that $b_{2}(Y)=0$. Besides, without loss of generality we may assume that $Y$ is connected. The arguments of the proof of Lemma 55 now apply (i.e. $Y$ may have finitely many isomorphism types, each having a hyperbolic fundamental group) and it follows that $\min _{Y} I(Y) \geq C_{\epsilon}^{\prime}$ where $C_{\epsilon}^{\prime}>0$ is a constant that only depends on $\epsilon$. Hence if (54) holds we have $I\left(X, X^{\prime}\right) \geq$ $\min _{Y} I(Y) \geq C_{\epsilon}^{\prime}$ and the result follows.

Suppose that inequality (54) is false, i.e. $I\left(X, X^{\prime}\right)<\min _{Y} I(Y)$, and consider a simplicial loop $\gamma: S^{1} \rightarrow X^{\prime}$ satisfying $\gamma \sim 1$ in $X$ and $|\gamma| \cdot A_{X}(\gamma)^{-1}<\min _{Y} I(Y)$. Let $\psi: D^{2} \rightarrow X$ be a simplicial spanning disc of minimal area. It follows from the arguments of Ronan [23], that $\psi$ is non-degenerate in the following sense: for any 2-simplex $\sigma$ of $D^{2}$ the image $\psi(\sigma)$ is a 2-simplex and for two distinct 2-simplexes $\sigma_{1}, \sigma_{2}$ of $D^{2}$ with $\psi\left(\sigma_{1}\right)=\psi\left(\sigma_{2}\right)$ the intersection $\sigma_{1} \cap \sigma_{2}$ is either $\emptyset$ or a vertex of $D^{2}$. In other words, we exclude foldings, i.e. situations such that $\psi\left(\sigma_{1}\right)=\psi\left(\sigma_{2}\right)$ and $\sigma_{1} \cap \sigma_{2}$ is an edge. Consider $Z=X^{\prime} \cup \psi\left(D^{2}\right)$. Note that $L(Z) \leq 0$. Indeed, since

$$
L(Z)=\sum_{e}\left(2-\operatorname{deg}_{Z}(e)\right)
$$

where $e$ runs over the edges of $Z$, we see that for $e \subset X^{\prime}, \operatorname{deg}_{X^{\prime}}(e) \leq \operatorname{deg}_{Z}(e)$ and for a newly created edge $e \subset \psi\left(D^{2}\right)$, clearly $\operatorname{deg}_{Z}(e) \geq 2$. Hence, $L(Z) \leq L\left(X^{\prime}\right) \leq 0$. On the other hand, $A_{X}(\gamma)=A_{Z}(\gamma)$ and hence $I(Z) \leq|\gamma| \cdot A_{X}(\gamma)^{-1}<\min _{Y} I(Y)$, a contradiction.

The main idea of the proof of Theorem 15 in the general case is to find a planar complex (a "singular surface") $\Sigma$, with one boundary component $\partial_{+} \Sigma$ being the initial loop and such that "the rest of the boundary" $\partial_{-} \Sigma$ is a "product of negative loops" (i.e. loops satisfying Lemma 56). The essential part of the proof is in estimating the area (the number of 2-simplexes) of such $\Sigma$.

Proof of Theorem 15. Consider a connected tight pure $\epsilon$-admissible 2-complex $X$ and a simplicial prime loop $\gamma: S^{1} \rightarrow X$ such that the ratio $|\gamma| \cdot A_{X}(\gamma)^{-1}$ is less than the minimum of the numbers $I\left(X^{\prime}\right)$ for all proper subcomplexes $X^{\prime} \subset X$. Consider 
a minimal spanning disc $b: D^{2} \rightarrow X$ for $\gamma=\left.b\right|_{\partial D^{2}}$; here $D^{2}$ is a triangulated disc and $b$ is a simplicial map. As we showed in Remark 53, the map $b$ is surjective. As explained in the proof of Lemma 56, due to the arguments of Ronan [23], we may assume that $b$ has no foldings.

For any integer $i \geq 1$ we denote by $X_{i} \subset X$ the pure subcomplex generated by all 2-simplexes $\sigma$ of $X$ such that the preimage $b^{-1}(\sigma) \subset D^{2}$ contains $\geq i$ twodimensional simplexes. One has $X=X_{1} \supset X_{2} \supset X_{3} \supset \cdots$. Each $X_{i}$ may have several connected components and we will denote by $\Lambda$ the set labeling all the connected components of the disjoint union $\bigsqcup_{i>1} X_{i}$. For $\lambda \in \Lambda$ the symbol $X_{\lambda}$ will denote the corresponding connected component of $\bigsqcup_{i \geq 1} X_{i}$ and the symbol $i=i(\lambda) \in\{1,2, \ldots\}$ will denote the index $i \geq 1$ such that $X_{\lambda}$ is a connected component of $X_{i}$, viewed as a subset of $\bigsqcup_{i \geq 1} X_{i}$. We endow $\Lambda$ with the following partial order: $\lambda_{1} \leq \lambda_{2}$ iff $X_{\lambda_{1}} \supset X_{\lambda_{2}}$ (where $X_{\lambda_{1}}$ and $X_{\lambda_{2}}$ are viewed as subsets of $X)$ and $i\left(\lambda_{1}\right) \leq i\left(\lambda_{2}\right)$.

Next we define the sets

$$
\Lambda^{-}=\left\{\lambda \in \Lambda ; L\left(X_{\lambda}\right) \leq 0\right\}
$$

and

$$
\Lambda^{+}=\left\{\lambda \in \Lambda \text {; for any } \mu \in \Lambda \text { with } \mu \leq \lambda, L\left(X_{\mu}\right)>0\right\} .
$$

Finally we consider the following subcomplex of the disk $D^{2}$ :

$$
\Sigma^{\prime}=D^{2}-\bigcup_{\lambda \in \Lambda^{-}} \operatorname{Int}\left(b^{-1}\left(X_{\lambda}\right)\right)
$$

and we shall denote by $\Sigma$ the connected component of $\Sigma^{\prime}$ containing the boundary circle $\partial D^{2}$.

Recall that for a 2-complex $X$ the symbol $f_{2}(X)$ denotes the number of 2-simplexes in $X$. We have

$$
f_{2}\left(D^{2}\right)=\sum_{\lambda \in \Lambda} f_{2}\left(X_{\lambda}\right)
$$

and

$$
f_{2}(\Sigma) \leq f_{2}\left(\Sigma^{\prime}\right)=\sum_{\lambda \in \Lambda^{+}} f_{2}\left(X_{\lambda}\right)
$$

Formula (56) follows from the observation that any 2-simplex of $X=b\left(D^{2}\right)$ contributes to the R.H.S. of (56) as many units as its multiplicity (the number of its preimages under $b$ ). Formula (57) follows from (56) and from the fact that for a 2 -simplex $\sigma$ of $\Sigma^{\prime}$ the image $b(\sigma)$ always lies in the complexes $X_{\lambda}$ with $L\left(X_{\lambda}\right)>0$.

Lemma 57. One has the following inequality

$$
\sum_{\lambda \in \Lambda^{+}} L\left(X_{\lambda}\right) \leq\left|\partial D^{2}\right| .
$$


See [10], Lemma 6.8 for the proof.

Now we continue with the proof of Theorem 15. Consider a tight $\epsilon$-admissible pure 2-complex $X$ and a simplicial loop $\gamma: S^{1} \rightarrow X$ as above. We will use the notation introduced earlier. The complex $\Sigma$ is a connected subcomplex of the disk $D^{2}$; it contains the boundary circle $\partial D^{2}$ which we will denote also by $\partial_{+} \Sigma$. The closure of the complement of $\Sigma$,

$$
N=\overline{D^{2}-\Sigma} \subset D^{2}
$$

is a pure 2-complex. Let $N=\bigcup_{j \in J} N_{j}$ be the strongly connected components of $N$. Each $N_{j}$ is PL-homeomorphic to a disc and we define

$$
\partial_{-} \Sigma=\bigcup_{j \in J} \partial N_{j}
$$

the union of the circles $\partial N_{j}$ which are the boundaries of the strongly connected components of $N$. It may happen that $\partial_{+} \Sigma$ and $\partial_{-} \Sigma$ have nonempty intersection. Also, the circles forming $\partial_{-} \Sigma$ may not be disjoint.

We claim that for any $j \in J$ there exists $\lambda \in \Lambda^{-}$such that $b\left(\partial N_{j}\right) \subset X_{\lambda}$. Indeed, let $\lambda_{1}, \ldots, \lambda_{r} \in \Lambda^{-}$be the minimal elements of $\Lambda^{-}$with respect to the partial order introduced earlier. The complexes $X_{\lambda_{1}}, \ldots, X_{\lambda_{r}}$ are connected and pairwise disjoint and for any $\lambda \in \Lambda^{-}$the complex $X_{\lambda}$ is a subcomplex of one of the sets $X_{\lambda_{i}}$, where $i=1, \ldots, r$. From our definition (55) it follows that the image of the circle $b\left(\partial N_{j}\right)$ is contained in the union $\bigcup_{i=1}^{r} X_{\lambda_{i}}$ but since $b\left(\partial N_{j}\right)$ is connected it must lie in one of the sets $X_{\lambda_{i}}$.

We may apply Lemma 56 to each of the circles $\partial N_{j}$. We obtain that each of the circles $\partial N_{j}$ admits a spanning disc of area $\leq K_{\epsilon}\left|\partial N_{j}\right|$, where $K_{\epsilon}=C_{\epsilon}^{\prime-1}$ is the inverse of the constant given by Lemma 56. Using the minimality of the disc $D^{2}$ we obtain that the circles $\partial N$ bound in $D^{2}$ several discs with the total area $A \leq K_{\epsilon} \cdot\left|\partial_{-} \Sigma\right|$.

For $\lambda \in \Lambda^{+}$one has $L\left(X_{\lambda}\right) \geq 1$ and $\chi\left(X_{\lambda}\right) \leq 1$ (since $b_{2}\left(X_{\lambda}\right)=0$ ); in particular, $f_{1}\left(X_{\lambda}\right) \geq f_{2}\left(X_{\lambda}\right)$. By Lemma 16 either

$$
3 \chi\left(X_{\lambda}\right)+L\left(X_{\lambda}\right) \geq \epsilon f_{1}\left(X_{\lambda}\right) \text { or } \quad 2 \chi\left(X_{\lambda}\right)+L\left(X_{\lambda}\right) \geq \epsilon f_{2}\left(X_{\lambda}\right) .
$$

Hence we have either

$$
4 L\left(X_{\lambda}\right) \geq 3 \chi\left(X_{\lambda}\right)+L\left(X_{\lambda}\right) \geq \epsilon f_{1}\left(X_{\lambda}\right) \geq \epsilon f_{2}\left(X_{\lambda}\right)
$$

or

$$
3 L\left(X_{\lambda}\right) \geq 2 \chi\left(X_{\lambda}\right)+L\left(X_{\lambda}\right) \geq \epsilon f_{2}\left(X_{\lambda}\right) .
$$

Since $L\left(X_{\lambda}\right) \geq 1$, both cases imply

$$
f_{2}\left(X_{\lambda}\right) \leq \frac{3}{\epsilon} L\left(X_{\lambda}\right)
$$

Summing up we get

$$
f_{2}(\Sigma) \leq \sum_{\lambda \in \Lambda^{+}} f_{2}\left(X_{\lambda}\right) \leq \frac{3}{\epsilon} \sum_{\lambda \in \Lambda^{+}} L\left(X_{\lambda}\right) \leq \frac{3}{\epsilon}\left|\partial D^{2}\right| .
$$


The rightmost inequality is given by Lemma 57 .

Next we observe that

$$
\left|\partial_{-} \Sigma\right| \leq 2 f_{2}(\Sigma)+\left|\partial_{+} \Sigma\right|
$$

Therefore, we obtain

$$
\begin{aligned}
f_{2}\left(D^{2}\right) & \leq f_{2}(\Sigma)+A \leq \frac{3}{\epsilon}|\gamma|+K_{\epsilon} \cdot 2 \cdot f_{2}(\Sigma)+K_{\epsilon}|\gamma| \\
& \leq\left(\frac{3}{\epsilon}\left(1+2 K_{\epsilon}\right)+K_{\epsilon}\right) \cdot|\gamma|
\end{aligned}
$$

implying

$$
I(X) \geq \frac{\epsilon}{3+6 K_{\epsilon}+\epsilon K_{\epsilon}} .
$$

This completes the proof of Theorem 15 .

\section{Acknowledgments}

This research was supported by an EPSRC grant.

The authors thank the anonymous referee for careful reading of the manuscript and making many helpful comments.

\section{References}

1. J. F. Adams, A new proof of a theorem of W. H. Cockcroft, J. London Math. Soc. 30 (1955) 482-488.

2. E. Babson, C. Hoffman and M. Kahle, The fundamental group of random 2-complexes, J. Amer. Math. Soc. 24 (2011) 1-28. See also the latest archive version arXiv:0711.2704.

3. E. Babson, Fundamental groups of random clique complexes, arXiv:1207.5028v2.

4. M. Bestvina and N. Brady, Morse theory and finiteness properties of groups, Invent. Math. 129 (1997) 445-470.

5. W. A. Bogley, J.H.C. Whitehead's asphericity question, in Two-dimensional Homotopy and Combinatorial Group Theory, eds. C. Hog-Angeloni, A. Sieradski and W. Metzler, LMS Lecture Notes, Vol. 197 (Cambridge Univ. Press, 1993), pp. 309-334.

6. W. H. Cockcroft, On two-dimensional aspherical complexes, Proc. London Math. Soc. 4 (1954) 375-384.

7. D. Cohen, A. E. Costa, M. Farber and T. Kappeler, Topology of random 2-complexes, J. Disc. Comput. Geom. 47 (2012) 117-149.

8. A. E. Costa and M. Farber, The asphericity of random 2-dimensional complexes, Random Structures and Algorithms 46 (2015) 261-273.

9. A. E. Costa, M. Farber and T. Kappeler, Topics of Stochastic Algebraic Topology, Proc. of the Workshop on Geometric and Topological Methods in Computer Science (GETCO), Electron. Notes Theor. Comput. Sci., Vol. 283 (Elsevier, 2012), pp. 53-70.

10. A. E. Costa and M. Farber, Geometry and topology of random 2-complexes, Isr. J. Math. 209 (2015) 883-927.

11. A. E. Costa, M. Farber and D. Horak, Fundamental groups of clique complexes of random graphs, Trans. LMS 2 (2015) 1-32. 
12. A. E. Costa and M. Farber, Random simplicial complexes, arXiv:1412.5805, to appear in Configuration Spaces - Geometry, Topology, and Representation Theory, eds. F. Callegaro, F. Cohen, C. DeConcini, E. M. Feichtner, G. Gaiffi and M. Salvetti (Springer, 2016).

13. A. Costa and M. Farber, Large random simplicial complexes, I, J. Topol. Anal. 8 (2016) 399-429.

14. A. Costa and M. Farber, Large random simplicial complexes, III; The critical dimension, arXiv:1512.08714.

15. S. Eilenberg and T. Ganea, On the Lusternik-Schnirelmann category of abstract groups, Ann. Math. 65 (1957) 517-518.

16. M. Gromov, Hyperbolic groups, in Essays in Group Theory, ed. S. M. Gersten (Springer, 1987), pp. 75-265.

17. N. Hartdfield and G. Ringel, Clean triangulations, Combinatorica 11 (1991) 145-155.

18. M. Kahle, Topology of random clique complexes, Disc. Math. 309 (2009) 1658-1671.

19. M. Kahle, Topology of random simplicial complexes: A survey, in Algebraic Topology: Applications and New Directions, Contemp. Math., Vol. 620 (Amer. Math. Soc., 2014), pp. 201-221.

20. N. Linial and R. Meshulam, Homological connectivity of random 2-complexes, Combinatorica 26 (2006) 475-487.

21. R. Meshulam and N. Wallach, Homological connectivity of random $k$-complexes, Random Structures Algorithms 34 (2009) 408-417.

22. M. Newman, A.-L. Barabási and D. J. Watts, The Structure and Dynamics of Networks (Princeton Univ. Press, 2006).

23. M. A. Ronan, On the second homotopy group of certain simplicial complexes and some combinatorial applications, Quart. J. Math. 32 (1981) 225-233.

24. S. Rosenbrock, The Whitehead Conjecture - An overview, Siberian Electronoc Math. Rep. 4 (2007) 440-449. 